\title{
Non-histone protein methylation: biological significance and bio-engineering potential
}

Roberto Di Blasi ${ }^{1,2}$, O. Blyuss ${ }^{3,4,5}$, John F Timms ${ }^{6}$, Daniel Conole ${ }^{7}$, Francesca Ceroni ${ }^{1,2}$, Harry J. Whitwell ${ }^{5,8} *$

${ }^{1}$ Department of Chemical Engineering, Faculty of Engineering, Imperial College London, London (UK)

${ }^{2}$ Imperial College Centre for Synthetic Biology, Imperial College London, London (UK)

${ }^{3}$ School of Physics, Astronomy and Mathematics, University of Hertfordshire, Hatfield (UK)

${ }^{4}$ Department of Paediatrics and Paediatric Infectious Diseases, Sechenov First Moscow State Medical University, Moscow (Russia)

${ }^{5}$ Department of Applied Mathematics, Lobachevsky State University of Nizhny Novgorod, Nizhny Novgorod (Russia)

${ }^{6}$ Department of Women's Cancer, EGA Institute for Women's Health, University College London, London (UK)

${ }^{7}$ Department of Chemistry, Imperial College London, London (UK)

${ }^{8}$ Department of Metabolomics, Digestion and Reproduction, Faculty of Medicine, Imperial College London, London (UK)

*Corresponding author: Harry Whitwell

Email: h.whitwell@imperial.ac.uk

Running Title: Protein methylation in cellular functions and bioengineering

Keywords: Protein methylation, bioengineering, post-translational modification (PTM), cellular regulation, proteomics 


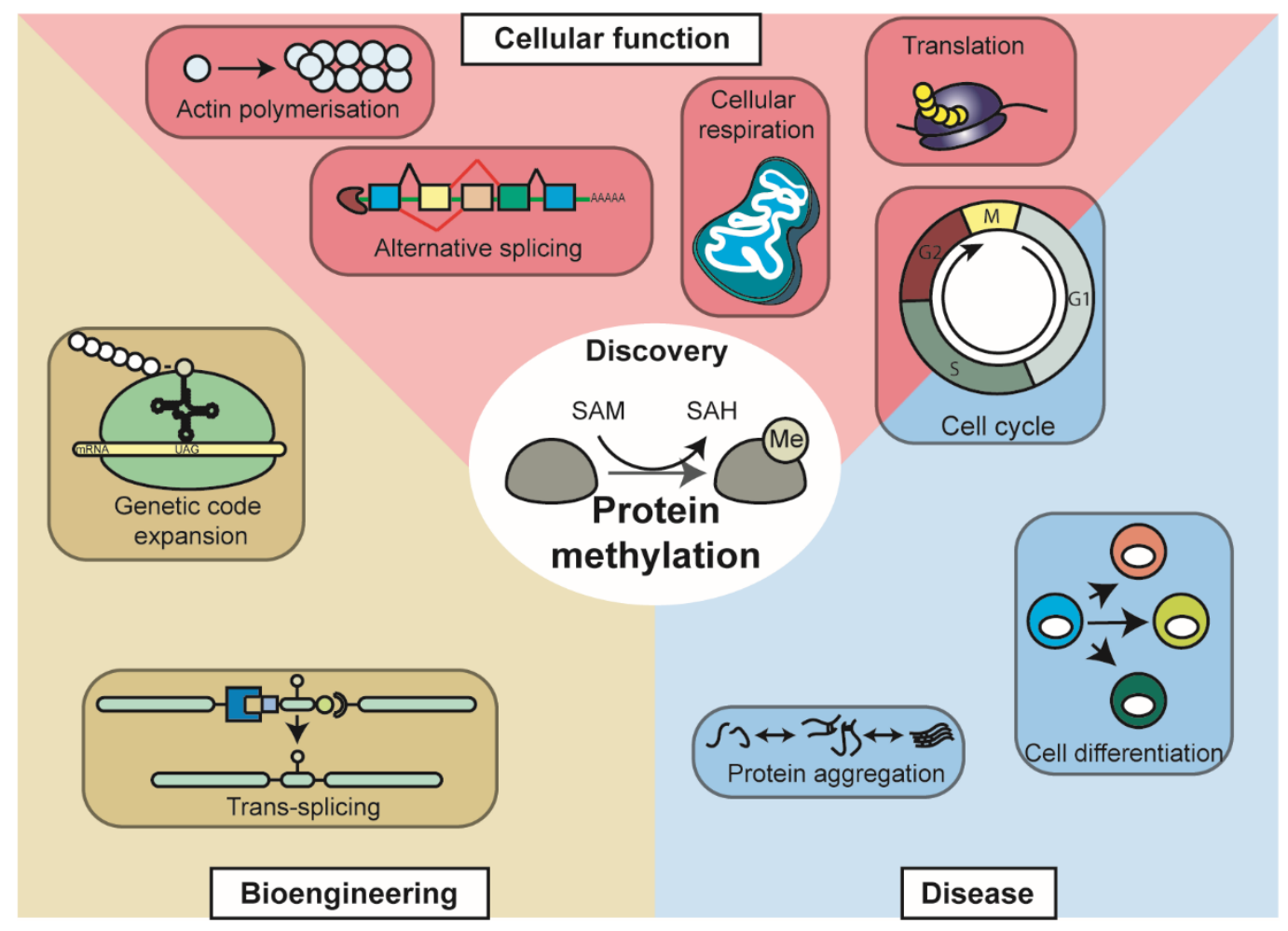

\begin{abstract}
Protein methylation is a key post-translational modification whose effects on gene expression have been intensively studied over the last two decades. Recently, renewed interest in non-histone protein methylation has gained momentum for its role in regulating important cellular processes and the activity of many proteins, including transcription factors, enzymes, and structural complexes. The extensive and dynamic role that protein methylation plays within the cell also highlights its potential for bioengineering applications. Indeed, while synthetic histone protein methylation has been extensively used to engineer gene expression, engineering of non-histone protein methylation has not been fully explored yet. Here, we report the latest findings, highlighting how non-histone protein methylation is fundamental for certain cellular functions and is implicated in disease, and review recent efforts in the engineering of protein methylation.
\end{abstract}

\title{
Introduction
}

In a study of the protein sequences of flagella in 1959, Ambler and Rees published the first biological example of a methyl-lysine containing protein, discovered in Salmonella typhimurium. ${ }^{1}$ Five years later, $\varepsilon$-N-methyl lysine was confirmed to be an abundant constituent of histone molecules ${ }^{2}$ and in 1968 , arginine methylation was identified from the action of "Protein Methylase I" - later designated the Protein arginine methyltransferase (PRMT) family. ${ }^{3-5}$ In 2004, the discovery of the first demethylase, LSD $1,{ }^{6}$ importantly showed that this post-translational modification (PTM) could be a dynamic feature of protein-mediated cellular regulatory pathways. Shortly after, jumonji domain containing 6 (JMJD6) was shown to have arginine demethylase activity against histone 3 Arg2 (H3R2) and histone 4 Arg3 $(\mathrm{H} 3 \mathrm{R} 3),{ }^{7}$ cementing the importance of dynamic histone modifications in biological regulation (Figure 1). JMJD6 also performs lysine hydroxylation (addition of hydroxyl group to $\delta$-C - lysyl-5hydroxylation), including on histone $\mathrm{H} 2 \mathrm{~A} / \mathrm{H} 2 \mathrm{~B}, \mathrm{H} 3$ and $\mathrm{H} 4$ and this modification can inhibit $\mathrm{N}$ methylation of the same residue, and vice-versa. ${ }^{8}$ Thus, its lysine-hydroxylation activity, at least in terms of epigenetic regulation, may be equally or more significant that its function as a demethylase.

Protein methylation refers to the addition of a $\mathrm{CH}_{3}$ moiety largely to the side chains of lysines and arginines, by methyltransferase enzymes with the universal methyl-cofactor S-adenosine L-methionine 
(SAM), which is itself converted to S-adenosylhomocysteine (SAH) (Figure 2). Lysine may be mono (me1)-, di (me2)- or tri (me3)-methylated, whereas arginine may be mono- (MMA), symmetrically di(SDM) or asymmetrically di-methylated (ASM) (Figure 1a). Other amino acids, such as Asn, Asp, Cys, Gln, Glu and Leu can be methylated, although in much lower abundance. ${ }^{9}$ Protein N-termini are also frequently methylated, and while just a few specific instances of histidine methylation have been identified in human cells, their presence on abundant proteins such as actin and myosin suggests strong biological relevance to the physiology of the cell.

Zhang et al. recently reported that protein methylation is a well-conserved mechanism in prokaryotes and eukaryotes although its biological function differed across species. ${ }^{9}$ Whilst much of the research relating to the function of protein methylation so far has focused on histone modifications, more recently, the essential role of non-histone protein methylation in the regulation of cellular pathways has become increasingly evident (Figure 1B). Sites of PTMs are highly conserved, ${ }^{10}$ and methylated residues are frequently proximal to other sites of modification. For example, in PhosphoSitePlus, ${ }^{11}$ over $60 \%$ of known methylated residues in non-histone human proteins are within 10 amino acids of a phosphorylation site and $>15 \%$ of lysine methylation competes with acetylation for occupancy of the same amino acid (Figure 1C). Mechanistically, the interplay between methylation and other PTMs (crosstalk) is highly important, either by working in combination (e.g. in the tumour suppressor P53(see below) or in competition, where PTMs compete for the same or mutually exclusive sites to elicit different responses (e.g. E2F, see below). Post-translational modifications that are proximal to each other can interfere directly with other protein-modifying reactions or protein binding within the same region. In this manner, complex crosstalk within histones is well documented but there are also important instances between methylated non-histone proteins and other PTMs (described below). As a chemical modification, protein methylation is relatively topologically small compared to other modifications (e.g. phosphorylation). It does not confer a significant change in charge distribution and so its functional mechanisms are likely to act via protein methylation "readers", direct inhibition of substrate binding (if in an active site or binding domain), or by affecting local hydrogen bonding. ${ }^{12-14}$

In this review, we will consider the currently available methodologies for detection of protein methylation and then present the latest work on the importance of non-histone protein methylation for the regulation of cellular functions and in disease. Finally, we will give an engineering perspective. Bioengineering tools have now become useful to systematically study histone and DNA modifications, and as platforms for genetic engineering. Similarly, we envision that deeper knowledge of non-histone protein modifications will pave the way for the development of a future toolbox for cell engineering. 


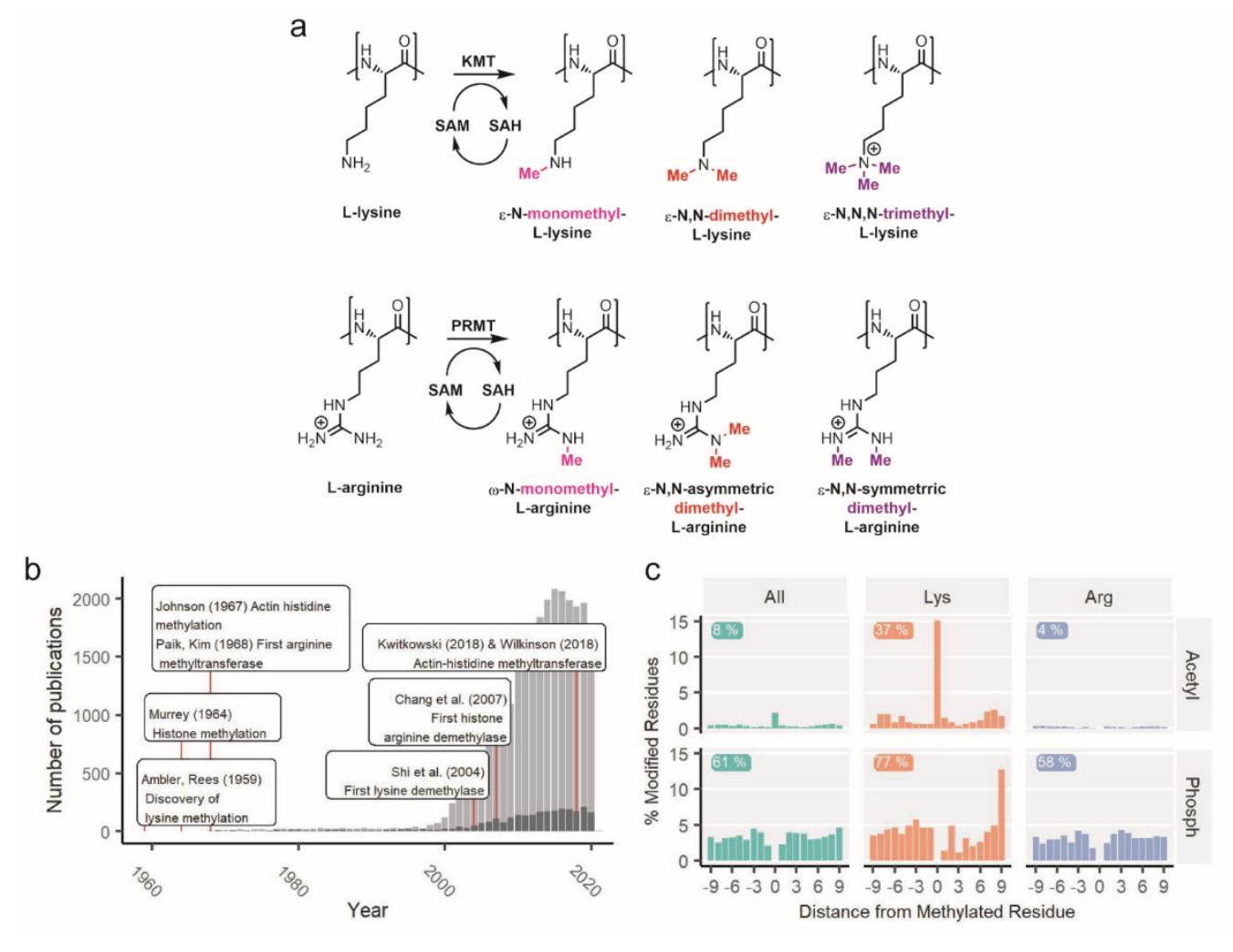

Figure 1 Protein methylation. a) Lysine methyltransferase (KMT) can add one, two or three methyl moieties to lysine side chains, resulting in monomethyl-lysine (pink), dimethyl-lysine (red), and trimethyl-lysine (purple) respectively (top panel). Protein arginine methyltransferase (PRMT) can add one or two methyl moieties to arginine side chains, resulting in monomethyl-arginine (pink), symmetrical dimethyl-arginine (purple), and asymmetric dimethyl-arginine (red) respectively (bottom panel). b) Number of publications from PubMed search of " protein methylation"[All Fields] OR "lysine methylation"[All Fields] OR "arginine methylation"[All Fields] OR "methyl lysine"[All Fields] OR "methyl arginine"[All Fields] OR "methyl cysteine"[All Fields]' (dark grey) or "histone methylation' (light grey), searched on 17/11/2020, with highlighted landmark developments in protein methylation understanding (red) c) Frequencies of methylated residues compared to their distances from a phosphorylated or acetylated residue (\% of total methylated amino acids from non-histone human proteins). Databases of methylation, phosphorylation and acetylation were downloaded from PhosphoSitePlus (v6.5.9.2) and filtered to include only residues with at least 3 sources of evidence. The percentage of methylated residues from human non-histone proteins (arginine (Arg, right), lysine (Lys, middle) or all residues (Cys, His, Lys, Leu and Arg, left)) with an acetylated (Acetyl, top) or phosphorylated (Phosph, bottom) within $+/-9$ amino acids. The total number of methylated residues with an acetylation or phosphorylation site with $+/-9$ amino acids is given in each plot.

\section{Role of protein methylation in the regulation of cellular functions and pathways.}

The past 2-3 decades have illuminated the many roles that non-histone protein methylation plays in the regulation of cellular pathways and functions. Indeed, methylation has been shown to play a key role in cell physiology, with many examples attesting to its importance in cell cycle progression and posttranscriptional/translational regulation, notwithstanding a plethora of cases where the fine tuning of specific proteins and cellular functions have been discovered. The mechanisms of action of non-histone protein methylation is broad. In a similar manner to histone methylation, they may act as docking points for methylation "readers" which bind to methylated proteins via specific domains, for example Tudor or malignant brain tumour (MBT) domains. The methylation dependent degradation of SOX2 (discussed in more detail below), is mediated through the recruitment of an E3-ubiquitin ligase 
following binding of L3MTBL3 (via its MBT domain) to methylated residues upon SOX2. Protein methylation may allosterically inhibit the binding of other post-translational modifiers (e.g. E2F - see below) resulting in complex regulation of the target protein (crosstalk), promote enzymatic activity (e.g. PP2A - see below) or, through the influence of altered hydrogen bonding (see FUS, discussed below).

Methylation is involved in the regulation of cell cycle.

Here we report the most recent findings on the role of protein methylation in regulating the cell cycle; a comprehensive review of protein arginine methylation in cell cycle regulation was published by Raposo et al.. ${ }^{15}$

The cell cycle is classically understood to be regulated through the combined action of cyclins and cyclin-dependent kinases (enzymes that add a phosphate as a PTM) which, when activated can steer the cell through cell-cycle check points. Polo-like kinases (PLKs) are also intrinsic to the cell cycle, affecting biological processes in the G1, S,G2 and M-phases. ${ }^{16}$ The regulation of kinases and their substrates must be highly regulated to maintain correct cell cycling. While regulation can be achieved at the transcriptional level, PTMs also play an essential role. As this complex system is further understood, so is the impact of multiple PTMs on the same substrate - i.e. PTM crosstalk.

PLK1 is involved in DNA repair and its activation is required for progression past the G2/S-phase boundary and cytokinesis. PLK1 is regulated temporally (gene expression) ${ }^{17}$ and chemically by phosphorylation of Thr210 (Thr210p) which is essential for its activation. PLK1 was recently identified as being methylated at a number of residues, including Lys209 ${ }^{18,19}$ and Lys413. ${ }^{19}$ Both these residues reside at conserved regions within the PLK1 protein, with Lys413 being adjacent to Trp414, a residue essential for substrate recognition and Lys209 being adjacent to Thr210 within the activating T-loop. ${ }^{19}$ Li et al. showed that K209me1 and Thr210p are mutually exclusive, and this has importance in both DNA damage response and mitotic progression. During the DNA damage response, PLK1 is inactivated by loss of Thr210p, preventing entry into mitosis. This inactivation is sustained by methylation at Lys209 by the methyltransferase EHMT/G9a which is also required for disassembly of DNA-repair machinery, although through unknown mechanisms. Interestingly, in the study by Feldman et al., an array-based approach identified SETD6 as being responsible for Lys209me1, but this activity was not replicated by Li et al. This is likely because, Li et al. who used immuno-precipitation to obtain cellular SETD6, may not have had sufficient quantity (or purity) required for its observable activity. Gene silencing of neither SETD6 nor G9a was able to completely ablate Lys209me1, and therefore it is likely that this redundancy is true.

As well as providing regulation of the G2/M checkpoint, PLK1 also functions during mitosis. Both Feldman and Li observed that methylated PLK1 delayed the rate of mitosis. Li further showed that methylation (and thus inactivation) of PLK1 blocked the disassociation of the cohesin complex during late metaphase, delaying the metaphase-anaphase transition. Feldman et al. demonstrated that SETD6 binds to PLK1 during mitosis, correlating with a peak in expression of SETD6, whose expression oscillates during cell cycling, unlike G9a which is expressed throughout the cell cycle. Thus, there is a multifaceted role for the regulation of PLK1 by methylation, both in terms of DNA-repair at the G2/M checkpoint and in regulation of mitosis. The timing of expression of G9a and SETD6 suggests a possible temporal relationship regulating the activity of PLK1.

A major regulator of cell-cycling and DNA damage-response, p53, is a master cellular regulator whose biological activity is driven by the crosstalk of different PTMs, including methylation. The C-terminal residues Arg333, 335, 337 and Lys370, 372, 373, 381 and 382 of human p53 are experimentally known to be methylation, with Lys373 and 382 also able to be acetylated (UniProtKB Entry version 287). A number of methyltransferases have been shown capable of methylating p53 in vitro (SMYD2, SETD7, PRMT5, EHMT1, EHMT2, KMT5A), although SETD7 does appear to have functional consequences in vivo, which may be due to other methyltransferases compensating for knock-outs in in vivo models. ${ }^{20}$ 
Cross talk between modified residues is important in regulating p53, it was shown recently that methylation within the C-terminal region of p53 promoted acetylation of Lys 117 which is required for p53-mediated apoptosis. In leukaemia, Growth Factor Independence 1 (GFI1) can be oncogenic, inhibiting p53-mediated apoptosis by recruiting LSD1 to de-methylate p53 in the C-terminal domain, thus inhibiting Lys117 acetylation. GFI1 is also a target of activated p53, suggesting a self-regulating feedback loop for $\mathrm{p} 53 .^{21}$

Another example of the role that protein methylation plays in cell cycle regulation comes from transcription factor E2F1. E2F1 plays a multifaceted role in cell proliferation and can behave either as tumour suppressor or as an oncogene, depending on the context. The biological activity of E2F1 is highly dependent on methylation and is regulated by the competition between the methyltransferases PRMT5 and PRMT1, whose methylation of E2F1 is mutually exclusive. ${ }^{22}$ Briefly, SDM of Arg111 and Arg113 on E2F1 by PRMT5 triggers cell-division, whereas ADM of Arg109 by PRMT1 leads to apoptosis. ${ }^{22}$ It was observed that cells treated with siRNA against PRTM1 had a hyper-proliferative phenotype and conversely, when treated with siRNA against PRTM5 were pro-apoptotic. Adjacent to the PRMT1 substrate (Arg109), is the binding domain for cyclin A, whose complexing with E2F1 promotes cell-cycle progression. Zheng et al. observed that binding of cyclin A impeded the ability of PRMT1 to methylate Arg109, permitting the binding of PRMT5 and its subsequent methylation of Arg111 and Arg113. More so, they identified that the transcriptional co-regulator p100 bound to methylated Arg111/113 by recognising the SDM modifications induced by PRMT5. Depletion of p100 resulted in increased apoptosis, suggesting that the proliferative phenotype induced by PRMT5 activity is mediated through p100. Interestingly, they observed that SDM Arg111/113-modified E2F1 had reduced ability to affect transcription, despite resulting in phenotypic changes. ${ }^{23}$ They elucidated that binding of p100 to E2F1 results in the alternative splicing of many E2F1 target genes, thus developing the mechanism by which PRMT5 methylation of E2F1 promotes cell-cycle advancement.

\section{Methylation regulates proteins involved in translational and post-translational regulation.}

Besides heavily impacting the function of numerous cell cycle regulators, non-histone protein methylation has also been shown to act on different proteins involved in the translational and posttranslational regulation of gene expression. We have already discussed the role protein methylation can play in alternative splicing through the activity of E2F1 and p100. Here we will comment on two further examples from the recent literature.

Elongation factor EF1A plays a crucial role in translation, recruiting aminoacyl-tRNAs to the ribosomal 80 s subunit, ${ }^{24}$ and is regulated at the post-translational level by a diverse set of methyltransferases. ${ }^{25}$ Małecki et $a l .{ }^{26}$ showed that the previously uncharacterised methyltransferase METTL21B can mono, di and trimethylate Lys 165 of eEF1A. They observed a high proportion of eEF1A carried Lys165me, but that there was variation in the degree of methylation between cancer and tissue-specific cell lines. When fibroblast cells were growth-inhibited through either contact-inhibition or serum-starvation, the proportion of Lys $165 \mathrm{me} 2$ increased from $\sim 10 \%$ to $\sim 30 \%$ in a reversible manner (e.g. upon supplementation of serum). Furthermore, these changes were mirrored by increased expression of METTL21B mRNA, showing that Lys165 methylation is dynamic and responds to changes in cellular state. The activity of METTL21B on eEF1A Lys165 was confirmed by Hamey et al. who further investigated the impact of METTL21B knock-out on protein expression. ${ }^{27}$ Knock-out of METT21B by CRISPR-Cas9 resulted in a similar profile to knock-out of EEF1AKMT1, which methylates eEF1A Lys79. However, gene-ontology enrichment analysis showed some unique effects of loss of METT21B, such as upregulation of ribosomal large subunit biogenesis and rRNA processing. It cannot be excluded that these changes are due to trans-effects of METT21B knock-out, rather than the direct impact of loss of eEF1A Lys165 methylation. This could be explored through eEF1A Lys165 mutants in future studies. 
SOX2 is a transcription factor that regulates embryonic stem cell renewal and pluripotency. Its expression is tightly controlled at both the transcriptional and translational level. Recently, methylation by SETD7 was shown to be an essential component of SOX2 regulation. Fang et al. ${ }^{28}$ demonstrated that methylation of Lys117 leads to degradation of SOX2 and that this is inhibited through the phosphorylation of the adjacent tyrosine (in mice). More recently, Zhang et al. ${ }^{29}$ demonstrated that Lys42me1 also leads to SOX2 proteolysis. Furthermore, knock-down of the lysine demethylase LSD1, which targets Lys42 and Lys117 causes a decrease in the abundance of SOX2 showing that regulation of SOX 2 by this mechanism is a dynamic and reversible process. Another level of regulation comes from the binding of the Tudor-domain containing methyl-reader PHF20L1, which competes for binding of methylated SOX2 by the E3-ubiquitin ligase-recruiting protein L3MBTL3, which binds to methylated residues via its MBT domain. ${ }^{30}$ Knock-down of LSD1 or PHF20L1 impairs pluripotency and self-renewal of murine embryonic stems cells which is rescued by the further silencing of L3MBTL3. ${ }^{30}$ This regulation of stem cell-pluripotency is important not only for disease, but it is conceivable that this complex combination of PTMs crosstalk and methylation-dependent protein degradation may provide a mechanism for precisely controlling cellular phenotype in future synthetic systems.

Mitochondrial activity relies on methylation of key proteins.

NDUFAF7 is an essential component of the mitochondrial respiratory complex I, with knock-outs being embryonic lethal in mice. ${ }^{31}$ Complex I is comprised of 44 subunits that form the first enzyme of the respiratory chain, generating an electron pair through the oxidation of NADH that subsequently moves down the electron transport chain. ${ }^{32}$ NDFUAF7 contains a methyltransferase domain, and its functionality is dependent on this activity. Rhein et al., and a year later Rendon et al., showed that NDUFAF7 is responsible for the SDM of R85 in the NDUFS2 subunit of complex I, which occurs during assembly of this macro-protein complex. ${ }^{31,33}$ Research has also pointed to the role of protein methylation in regulating cellular respiration through glucose sensing and tuning of mitochondrial activity. Małecki et al. reported the functional characterisation of the ANT-KMT (formally FAM173A) and its paralogue ATPSc-KMT (formally FAM173B) which were found to tri-methylate ATP synthase c-subunit (ATPSc) at Lys43 and adenine nucleotide translocase (ANT) at Lys52 respectively. ${ }^{34,35}$ Knock-out models of either enzymes affected respiration rate. Specifically, methylation of ATPSc is required for its correct incorporation into the ATP-synthase complex and to maintain ATP production, whereas ANT methylation decreases it. The degree of methylation within the mitochondrion, and its conservation across species, suggests some large significance in fine tuning respiratory output. ${ }^{36}$

In response to glucose starvation, sirtuins (SIRT) regulate cellular metabolism. In fasted mice provided with intraperitoneal glucose, SIRT7 methylation at Arg388 by PRMT6 increases in a time-dependent manner, inhibiting its histone deacetylase activity. SIRT7 regulates genes promoting mitochondrial biogenesis and inhibition of PRTM6 causes a loss in mitochondrial mass. In low glucose, the glucosesensing AMP-activated protein kinase (AMPK) is activated and inhibits PRTM6 downstream, blocking SIRT7 methylation and promoting its histone deacetylase activity, preventing mitochondrial biogenesis. ${ }^{37}$ This is an interesting finding demonstrating that protein methylation can be a responsive mechanism for signal transduction from external stimuli.

Actin methylation is essential for correct smooth muscle contractility.

Actin is highly expressed in all eukaryotic cells. It is present in both monomeric and polymeric forms that are the basis of the cytoskeletal system, important for cell motility, structural integrity, and muscle contractility. Actin is regulated by many PTMs, including methylation. Histidine methylation in actin was first discovered in 1967 by Johnson et al. who showed it to be conserved through mammalian, fish and bird species. ${ }^{38}$ Despite it being known to be functionally important, possibly by affecting interdomain motility and sensing of ATP/ADP state during actin polymerisation, ${ }^{39,40}$ it was only very recently that the responsible methyltransferase was identified as SETD3. ${ }^{41,42}$ 
The comprehensive function of SETD3 is not yet fully elucidated, whilst it is involved in several biological pathways, only actin has been conclusively identified as a substrate. Actin His 73 methylation is conserved across all species except yeast, owing to the lack of a SETD3 homologue. ${ }^{42}$ SETD3 had been reported to be a histone methyltransferase, however the masses observed by MS in this study were not explained by the addition of a methyl group and the finding was not repeatable by Wilkinson et al using western blotting, ${ }^{42}$ or by Guo et al. ${ }^{43}$ who both demonstrated little or no lysine methyltransferase activity. Rather, both these groups showed it to be an actin-specific histidine methyltransferase monomethylating actin at His $73 .{ }^{41-44}$

Actin His73me stabilises filamentous actin, with a loss of methylation being shown to increase depolymerization, Kwiatkowski showed that in the absence of SETD3, a concomitant decrease in filamentous actin is observed. ${ }^{41}$ Whilst this shows a physiological role for His 73 methylation, SETD3${ }^{1-}$ mice are viable, although have several pathologies. These include skeletal muscle myopathy, abnormal cardiac electrocardiogram, mildly decreased lean mass and (in females) smaller litter sizes and dystocia. ${ }^{42}$ Upon exploring the mechanism behind dystocia, Wilkinson et al. showed that SETD3depleted primary human myometrial cells were less responsive to oxytocin and endothelin-1 for inducing contraction and this was reversible upon re-expression of SETD3, implying His73me is important for muscle contractility. ${ }^{42,43}$ Prior to the identification of actin His73 as a substrate of SETD3, Abaev-Schneiderman et al. used proteomics data analysis to study the role of SETD3 in DNA damage response/apoptosis. ${ }^{45}$ They reported that among the overexpressed proteins, candidates related to the DNA damage response were enriched, suggesting either a role for this enzyme beyond its methyltransferase activity, or having substrates as-yet unknown, although some proteomics changes could be in response to a reduction in actin polymerisation. The expression of SETD3 varies across the cell-cycle, peaking in S-phase. ${ }^{46}$ It is perhaps suprising then that SETD3 expression is minimal during M-phase as actin polymerisation is particularly important during the extensive cytoskeletal rearrangments during mitosis and cytokinesis. Nevertheless, SETD3 appears to be involved in cellcycle regulation as loss of expression attenuates cell-cycling in liver carcinoma cell lines. Expression of SETD3 protein is elevated in liver tumours, with expression correlating with severity. Interestingly, Cheng et al. showed mRNA expression of SETD3 was slightly decreased in liver tumours, indicating that regulation of SETD3 is predominantly at the protein level.

\section{Role of protein methylation in disease}

Compared to histone methylation, there is a relative paucity of knowledge about the role of non-histone protein methylation in disease aetiology and progression. However, aberrant expression and/or mutation of several protein methyltransferases and demethylases is apparent in different pathologies, particularly cancer. For example, PRMT5 is overexpressed in many tumour types, appears to play an oncogenic role and is a potential therapeutic target. ${ }^{47} \mathrm{EZH} 2$ is also consistently overexpressed in a broad range of carcinomas and tumour-derived cell lines with its knock-down substantially reducing cancer cell proliferation. Although best known for trimethylation of histone H3K27, EZH2 has also been reported to methylate the non-histone protein, GATA-binding protein 4 (GATA4), repressing its transcriptional activity. ${ }^{48}$ Methylation may also modulate protein-protein interactions, for example, through chromodomain binding to dimethylated lysine, or can directly or indirectly alter other PTMs such as phosphorylation and ubiquitination to affect enzyme affinity, protein stability, promoter binding and subcellular localization. Examples of this are the EZH2-dependent methylation of RAR-related orphan receptor alpha $(\mathrm{ROR} \alpha)$, marking it for ubiquitin-dependent degradation ${ }^{48}$ and lysine methylation of MAP3K2 by SMYD3, which enhances Ras-driven cancer by blocking its interaction with protein phosphatase 2A (PP2A); a key negative regulator of MAPK signalling. ${ }^{49}$

A growing number of non-histone proteins important in cancer have been shown to be modulated by methylation, including $\mathrm{p} 53,{ }^{50} \mathrm{RB} 1,{ }^{51}$ VEGFR $1,{ }^{52}$ PARP1, ${ }^{53}$ FLT3, ${ }^{54}$ and heat-shock proteins. ${ }^{55}$ Evidence of the involvement of protein methylation in disease has prompted the development of drugs 
targeting the enzymes involved, particularly for cancer treatment. ${ }^{56,57}$ As an example, the DOT1L inhibitor, pinometostat showed modest efficacy in a phase I trial of advanced acute leukemia. ${ }^{58}$ However, pinometostat was also reported to increase susceptibility to viral infection, ${ }^{59}$ and DOT1L may have other non-oncogenic roles, for example, it appears to play a protective role in UV-induced melanomagenesis. ${ }^{60}$ Thus, if we are to fully understand the effects of existing drugs or to direct the development of new more effective therapies, it is imperative that we improve our knowledge of methyltransferase and demethylase substrate specificities, the biological pathways in which they act and how methylation is specifically dysregulated in disease. As our knowledge of non-histone methylation increases, it is likely that new drug targets will be identified and strategies to develop drugs against them should involve monitoring of specific methylation as a pharmacodynamic readout. An example here is FLT3 methylation by PRMT1 in acute lymphoblastic leukaemia, where inhibition of cell viability with a PRMT1-specific inhibitor negatively correlated with FLT3 methylation levels. ${ }^{54}$

Methyl transferases and demethylases are also potentially useful diagnostic and prognostic tumour markers by virtue of their altered expression or mutation in cancer. Their effective use as markers should consider their cellular localisation and activity in different cellular compartments which has been demonstrated for PRMT5. ${ }^{61}$ Their use as markers will most likely be restricted to tumour tissue specimen analysis, given that these enzymes are strictly cellular proteins lacking a signal sequence and therefore unlikely to be secreted into the circulation at any significant level. Thus, their use as sensitive, non-invasive biomarkers is doubtful. Conversely, the use of specific substrate methylation as a readout may provide useful cancer biomarkers, as suggested for FLT3 and HSP70 ${ }^{54,62}$ The analysis of specific methylation events may also provide biomarkers that are predictive of response to methyltransferase inhibitors. This would allow patient stratification for personalised medicine and improved capacity to treat disease.

\section{Methyltransferase Dependency in MTAP ${ }^{-/}$Cancer}

Upon a methyltransferase reaction, SAM is converted to S-adenosylhomocysteine (SAH), which is metabolised into homocysteine, methionine and back into SAM through the methionine cycle. This cycle is intertwined with other metabolomic pathways, sharing intermediates with the folate pathways and polyamine pathways (Figure 2). In polyamine synthesis, SAM is metabolised into methylthioadenosine (MTA), which is toxic to the cell in high concentrations and therefore is rapidly metabolised by S-methyl-5'-thioadenosine phosphorylase (MTAP) into methionine where it re-enters the methionine cycle. The MTAP gene is located within 9p21, also shared by the tumour-suppressor gene p16, which is one of the most frequent chromosomal deletions in cancer. Given the proximity of MTAP to p16, the incidence of MTAP deletion is also very high $(>60 \%)$, particularly in mesothelioma. ${ }^{63}$ MTAP deletion results in increased cellular MTA, which can selectively inhibit the methyltransferase PRMT5, sensitising MTAP $^{-/}$cells to PRMT5 inhibition and leading to cell death ${ }^{64-}$

${ }^{66}$ - possibly via the PRMT1/PRMT5 axis as discussed earlier, but also potentially via p53-mediated apoptosis. Strobl et al. found than upon inhibition of PRMT5, shorter, exon-skipped splicoforms of MDM4, a regulator of p53 activity, were transcribed, as well as observing increased p53 expression. ${ }^{67}$ However, while PRMT5 inhibition decreases cell survival in $\mathrm{MTAP}^{-/}$cells, it also reduced the viability of cells that retained MTAP expression. Furthermore, Strobl et al showed that the inhibition of PRMT5 supressed the cellular expansion of tumour-supressing CD8+ T-cells. There is substantial overlap between the substrates of PRMT1 and PRMT5, indeed, silencing of PRMT1 also sensitises cells to PRMT5 inhibition. ${ }^{68}$ Therefore, it may be that cancer cells are able to adapt to PRMT5 inhibitor therapeutics through redundancy of substrates for PRMT1. Further research in this area should explore what pathways PRMT5 activity is critical in in MTAP $^{-/-}$cancer to better understand the cellular adaptation to loss of MTAP and develop more tailored therapeutics. 


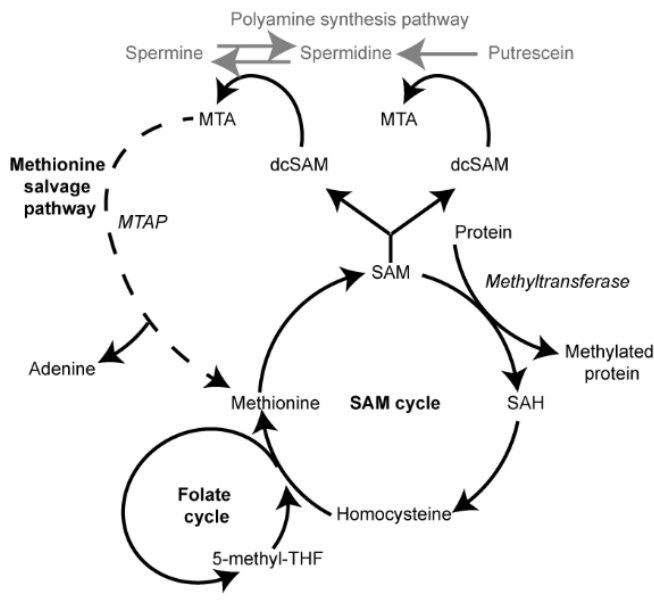

Figure 2 Methionine and SAM synthetic pathways. The SAM Cycle maintains endogenous levels of S-adenosylmethionine (SAM) and meshes with numerous metabolic cycles. Upon reaction with a substrate, catalysed by methyltransferases, SAM is converted to S-adenosylhomocysteine (SAH) which, through the SAM cycle, re-acquires a methyl-group from 5 methyl-tetrahydrofolate 5-methylTHF), a product of the Folate cycle. SAM may also be directed into the polyamine pathways, following decarboxylation (decarboxylated SAM, dcSAM) and converted into methylthioadenosine (MTA). MTA is toxic to the cell and is rapidly shuttled through the methionine salvage pathway (MSP) back into methionine. Critical to this step is the activity of methylthioadenosine phosphorylase (MTAP), which removes the adenine group from MTA on route to forming the intermediate methylthioribose-1phosphate (not shown), a precursor of methionine.

\section{Protein Methylation in Neurological Disease}

Alzheimer's disease (AD) is a neurodegenerative disease characterised by the development of neurofibrillary tangles and accumulation of the amyloid- $\beta$ protein. Neurofibrillary tangles are caused by the agglomeration of hypo-phosphorylated Tau protein which, under healthy physiological conditions, regulates the length of microtubules. Hyper-phosphorylation of Tau disrupts its binding to microtubules, promoting Tau-Tau interactions and the development of neurofibrillary tangles. ${ }^{69}$ Phosphorylation of Tau proteins is regulated in part by the activity of the phosphatase PP2A. O-linked methylation of Leu309 within the catalytic subunit of PP2A is required for its effective phosphatase activity. ${ }^{70}$ Loss of Leu309 methylation has been observed in $\mathrm{AD}$ and a role has also been highlighted in cancer. ${ }^{71}$ Methylation of this site is controlled by leucine carboxyl-methyltransferase 1 (LCMT1) and the demethylase protein phosphatase methyl-esterase (PME1) whose expression are reciprocally correlated in AD neuronal tissue. ${ }^{72}$ Tau protein has diverse PTMs, including lysine mono- and dimethylation which were identified on 13 distinct tryptic peptides. ${ }^{73,74}$ Whilst the function of Tau methylation in the context of other PTMs is unknown, it has been shown that it can reduce Tau fibril formation $^{74}$ and in a small cohort, accumulation of Tau methylation correlated with age and protection from $\mathrm{AD} .{ }^{75}$ In familial amyotrophic lateral sclerosis (fALS), aggregation of the RNA-binding protein FUS is a pathological feature that impairs protein synthesis. Whilst several mutations have been shown to promote aggregation, gel-promoting interactions between tyrosine and arginine residues are hindered by PRMT1-mediated arginine methylation ${ }^{76}$, reducing the strength of $\pi$-interactions between the residues. ${ }^{13}$ Similarly, in AD, formation of Tau fibrils is mediated by $\pi$-stacking of arginine via its guanidinium side chain. ${ }^{14}$ Therefore, it is possible that a common mechanism may exist that reduces $\pi$ stacking through arginine methylation.

\section{Challenges and Strategies for Studying the Protein Methylome.}


The global discovery of methylated proteins, or methyl-transferase specific substrates, is often performed by mass spectrometry (MS), which requires enrichment of methylated targets and comprehensive cross-validation of engineered methyltransferase-specific co-factors ${ }^{77}$ to overcome false-positive identifications. MS can identify many proteoforms in a single run, and be optimised to quantify, with high accuracy, many methylated peptides in a short time, making the technology a possibility for clinical applications. ${ }^{78}$ Array-based technologies are an alternative to MS, but these are limited by poor antibody sensitivity/specificity, limited proteoform presence on the chip/array and a lack of biological context of the methylation reaction. ${ }^{79}$

Standard MS workflows applied to studying protein methylation results in intolerably high false discovery rates due to the number of possible isobaric non-methylated and methylated peptides, and the methylation of acidic residues arising when methanol/ethanol is present in sample preparation buffers. ${ }^{80}$ Whilst it is possible to adjust some mass spectrometers to provide signatures indicative of arginine methylation (e.g ${ }^{81,82}$ ), these are not appropriate for global proteomics studies. The most robust approach is to use isomethionine methyl-stable isotope labelling of amino acids in cell culture (iMethylSILAC) ${ }^{83}$, a progression of heavy-methyl SILAC (hmSILAC) ${ }^{84}$ Cells are cultured with methionine containing normal or an isotopically-heavy $\left({ }^{13} \mathrm{CD}_{3}\right)$ methyl group. Upon cellular internalisation, methionine is converted to SAM by the methionine cycle (Figure 2) and the action of methyltransferases adds $\mathrm{CH}_{3}$ or ${ }^{13} \mathrm{CD}_{3}$ to protein-backbones. Differentially treated cells can then be analysed simultaneously by MS, and true methyl-groups are identified by the present of both heavy and light versions. To aid with analysing such data, open-source programs have recently been developed, MethylQuant ${ }^{85}$ and hmSEEKER ${ }^{86}$ though both are limited in their compatibility with analytical pipelines. The use of methyl site-predictive tools, such as DeepRMethylSite which uses convolutional neural networks, may also be helpful for identifying methyl arginine. ${ }^{87}$

Enrichment of methylated peptides prior to MS is essential to capture low stoichiometry modifications. However, whilst antibodies against arginine methylation can return thousands of methylated $\operatorname{sites}^{88}$, antibodies against lysine methylation are less successful. ${ }^{89}$ For example, in Guo et al. ${ }^{88}$ antibodies were raised against random methylated hexameric oligopeptides and selected with comprehensive crossvalidation. They were able to identify $\sim 2000$ methyl arginine sites, but only $\sim 200$ methyl lysine sites, despite the relative proportions of these modifications likely to be far more equitable. ${ }^{11}$ Fractionation by strong cation exchange (SCX), which exploits the increase in charge of methylated peptides with a missed-tryptic cleavage, can be effective for increasing the overall number of methyl-lysine sites, particularly when combined with immuno-affinity enrichment, with a 4-fold increase in siteidentification achieved by the Garcia group by this approach..$^{89,90}$ The combination of fractionation and immuno-affinity enrichment can identify many thousands of methylation sites. ${ }^{91,92}$ Hartel et al. benchmarked these two approaches and showed that these strategies are largely orthogonal and so both are required for comprehensive identification of methylated residues, with SCX identifying a greater proportion of highly methylated (>2 methyl groups) peptides compared to immunoaffinity. ${ }^{93}$ However, SCX enrichment, which exploits the loss of tryptic cleavage at methylated residues, is less effective for mono-methyl lysine and better approaches are required.

Studies of "global" methylation typically focus on arginine, lysine or both. However, it should be considered that this is by no means inclusive of all protein methylation - many other amino acids been identified from hmSILAC experiments. ${ }^{9}$ Therefore, the possibility of non-arginine/lysine methylation should be considered in studies of global methylation, as it can be important. Histidine methylation of actin, first identified in $1967,{ }^{38}$ is an important modification and carboxy-methylation of leucine (Leu309) of protein phosphatase 2A is implicated in Alzheimer's disease. ${ }^{94,95}$ Therefore, whilst excellent progress has been made in identifying and validating methylated proteins, better enrichment strategies are needed to identify them, in particular for non-arginine methylation. For example, a methyl-lysine prediction tool (MethylSight) suggested 70 new methylation sites on histone-proteins alone, $90 \%$ of which were experimentally validated. Expanding this tool into the whole proteome 
reveals approximately 10,000 proteins with novel putative methyl-lysine sites, with particular enrichment for proteins involved in regulating the cell cycle and DNA damage response. ${ }^{96}$

\section{Applications in biological engineering}

We have so far discussed the role of non-histone protein methylation in cellular processes and disease, and highlighted that novel information is now becoming available. We recognise that, as with epigenome editing, non-histone protein methylation represents a powerful tool for biological engineering applications. Indeed, PTMs, protein methylation among them, are attractive from an engineering perspective for the quick response they can elicit from their targets. By using PTMs, cells can quickly and cheaply regulate gene expression or enzymatic function, maintaining the ability to dynamically react without extra gene expression cost for the cells. This is a desirable feature for being able to adapt to rapidly changing environmental needs. As we highlight in this section, biological engineering takes advantage of natural PTMs to develop tools that can aid a better understanding of natural processes and regulation, but also to design and improve our ability to engineer living cells. Compared to chemical inhibition, or the incorporation of static chemical modifications (e.g. through the use of genetic code expansion, see below), biological engineering can provide dynamic systems that are incorporated into regulatory cascades, taking advantage of endogenous special and temporal regulation.

\section{Considerations for adopting chemical approaches for the characterisation of protein methylation}

Biological engineering approaches to study protein methylation typically employ modulation of gene expression through CRISPR-Cas9 gene editing tools, or the overexpression of fusion proteins. These methods are highly useful to deconvolute the significance of particular protein methylation events, however due to the engineered nature of these approaches they tend to obscure the endogenous context of the cell, therefore inhibiting the ability to study protein methylation under disease relevant cellular contexts.

On the other hand, small molecule chemical probes designed using information garnered from the structure, binding mode, and biochemical reactivity of methyltransferase co-factors (such as SAM or $\mathrm{SAH}$ ) can offer significant insight into the methyltransferase activity and substrate profiles in a native context. Moreover, chemical approaches for understanding the molecular mechanism for disease are more easily translated into therapeutics than bio-engineering systems, in the form of orally bioavailable small molecule drugs, although it is possible that some bio-engineered products could be realised as biosimilars for intravenous administration. Broadly, the application of co-factor analogues to study methyltransferase enzymes is considered a more nuanced tool than genetic approaches (such as mutagenesis), as they can be applied in a concentration and time-dependent manner, and used to monitor changes in methyltransferase activity in response to externally applied cellular stresses aimed to mimic certain diseases. ${ }^{97}$

Methyltransferases are a large and diversified class of enzymes that target many protein substrates. ${ }^{98}$ The selectivity of methyltransferase chemical probes is dependent on the methyltransferase substrate sub-class that is being targeted. However, the ability to discriminate the substrates for just one enzyme within a class remains challenging. Consequently, chemical probes generally lack the targeting ability (offered by bioengineering approaches) to identify specific methyltransferase substrates and therefore attribute protein function and disease relevance.

It is likely that the most successful efforts towards understanding the complex landscape and significance of non-histone protein methylation to disease will leverage a combination of approaches. For example, a promising chemical genetics method, termed 'bump and hole', which employs both pharmacology and bioengineering tools, has been developed to elucidate substrate profiles for 
individual methyltransferases. In this approach, both the wild-type (WT) enzyme and its natural cofactor are specifically rewired to construct a "pair", where the enzyme is bioengineered to generate a 'hole' in its active site, while the cofactor is elaborated with large substituent that acts as a 'bump' that will only fit into that 'hole.' Consequently, transfection of the mutant enzyme and treatment with the bump probe in cells allows determination of the substrates specific to this enzyme. For the study of methyltransferases, this approach was first applied to a protein arginine methyltransferase, whereby a single E117G mutation in the cofactor-binding pocket selectively accommodated a $\mathrm{N}^{6}$ benzyl SAM analogue, offering 67-fold selectivity over the WT system..$^{98}$ In addition to its application towards other transferase enzymes, such as kinases and acetyltransferases, ${ }^{99}$ the 'bump and hole' approach has since been widely adopted for the study of methyltransferase substrate profiles, ${ }^{97}$ and should continue to be a useful complementary approach to deconvolute the emerging importance of non-histone protein methylation in disease.

Some other current challenges with the design of methyltransferase chemical probes to understand protein methylation biology is their poor biological stability and cell permeability. Due to their hydrophilic properties, exogenous SAM/SAH and or their chemical probes do not easily enter the cell by passive diffusion, and even if one can design a more lipophilic analogue, these probes can in some cases be deactivated by altered $\mathrm{pH}$ in certain cellular compartments or hydrolytic enzymes. ${ }^{100}$ While in cellulo synthesis of SAM analogues can be achieved by overexpressing methionine adenosyltransferase (MAT) enzymes, ${ }^{100}$ further solutions to address these issues in complex and native biological systems are greatly needed.

\section{Foundational Work - Engineering Histone Methylation}

Engineering tools have been developed as a powerful approach to regulate gene expression via histone PTMs. For instance, Haynes and colleagues, ${ }^{101-103}$ have focused on the design and testing of synthetic histone-binding Polycomb-based transcription factors to regulate gene expression on demand. Another approach to achieve epigenetic regulation is to use customised DNA-binding proteins fused to the catalytic domains of histone modifiers (Figure 3a). This allows histone modifiers to be targeted to user-defined locations for gene expression modulation. In addition, advances in CRISPR technology allow the development of fusion proteins where the catalytic domains of transcriptional repressors and activators can be fused to dCas 9 for targeted control of gene expression in eukaryotes. ${ }^{104}$ The manipulation of histone epigenetic signatures can also be achieved through engineering histone methylation. For example, G9a, SUV39H1, EZH2, ${ }^{105}$ LSD1, ${ }^{106}$ DOT1L, PRMD9 ${ }^{107}$, and $\mathrm{KRAB}^{108}$ have all been repurposed for engineering tailor-made histone modifications, and in most cases have been useful in advancing our knowledge of epigenetic regulation of gene expression.

One route suited to in vivo studies is to exploit genetic code expansion, mediating the incorporation into proteins of non-canonical and/or post-translationally modified amino acids. This is done by chemically charging frameshift and amber suppressor tRNAs with the modified residues, so that they could then be used to introduce the desired modifications, following appropriate engineering of the protein-coding sequence. Schultz ${ }^{112,113}$ and $\mathrm{Chin}^{114,115}$ pioneered this technique achieving incorporation of $\mathrm{N}$-methyl lysine into histone proteins. More recently, Tokuda et al. used $E$. coli cell free protein synthesis to introduce methyl-lysines at specific positions of histone $\mathrm{H} 3$ and calmodulin by chemically charging frameshift and amber suppressor tRNAs with the methyl-modified residues. ${ }^{116}$ Despite this being applied to a histone protein, the technique could be applied to any protein of interest. Yanagisawa et al. engineered histone $\mathrm{H} 3$ with monomethyl-Lys at five positions simultaneously. They used $E$. coli RFO strains that are unable to produce the RF1 factor (and thus do not do not trigger termination of translation in response to the engineered amber codon), as well as cell-free protein synthesis-based assays, to introduce Boc-protected monomethyl-Lys at amber 
codons, thanks to an engineered pyrrolysyl-tRNA synthetase-tRNA ${ }^{\text {pyl }}$ pair. ${ }^{117}$ The power of genetic code expansion is well-known to bioengineers, and this is reflected in the recent surge of synthetic genomic platforms with repurposed stop codons. Although this technology has been widely used to introduce targeted post-translational modifications on histone proteins, it represents a valuable asset also when non-histone PTMs engineering is desired (Figure 3b).

Lessons learned from histone methylation can inform the engineering of non-histone protein modification

While instructing cells to acquire histone PTMs at specific genomic locations has become relatively straightforward thanks to our current ability to engineer DNA-binding specificity, the imposition of specific PTMs on non-histone proteins still proves very challenging due to both the lack of background knowledge and specific tools.

Much progress in site-specific histone modification has come from the fusion of dCas9 and active domains from methyltransferases or other PTM-mediating enzymes (Figure 3a). Similarly to Cas9 specificity for DNA target sites, proteins can be engineered to specifically bind peptides present in other proteins via protein-peptide interactions (e.g. herteromeric coiled-coil dimers can be designed based on charge-mediated interaction rules). ${ }^{109,110}$ Tools have been developed that can identify peptide binding sites serving as docking platforms for cognate protein enzymes. Improvement in our knowledge on the specificity of peptide-protein binding, their design and prediction could enable us to engineer PTMs at specific residues using fusion between a peptide-binding domains and catalytic domains. De-novo peptide design will then enable us to regulate the PTM switch on and off status with precision. ${ }^{111}$

Khoo et al., proposed that split intein-mediated trans-protein splicing can be a tool to engineer protein methylation, and other PTMs in vivo. ${ }^{118}$ Inteins are autocatalytic peptides that are translated as internal parts of longer polypeptide chains, and are able to mediate their self-excision, joining the flanking amino acid residues through a peptide bond. Split inteins are a particular class of inteins where two separate peptide stretches, belonging to two different polypeptide chains mediate their joining, thus catalysing the protein splicing of the two polypeptides in trans. Khoo et al. used split intein pairs to mediate the splicing amongst the heterologous $\mathrm{C}$ - and $\mathrm{N}$-protein fragments, and the chemically synthesised central fragment carrying the desired modification(s). In this way, it was possible to reconstruct the full-length modified protein directly inside the cell, after intracellular administration of the synthetic peptide. The authors succeeded in modifying cytosolic as well as membrane proteins, both in their intracellular, and extracellular domains in eukaryotic cells. Previously, David et al. ${ }^{119}$ reported on the engineering of intein pairs where the $\mathrm{N}$-terminal fragment of a split intein is fused to $\mathrm{H} 2 \mathrm{~B}$ while the $\mathrm{C}$-terminal fragment is fused to a synthetic probe bearing the modification of interest. Upon delivery of the $\mathrm{C}$-intein-probe system in cells engineered to express the $\mathrm{N}$-intein-H2B fusion, reaction of the two intein pairs led to the desired histone modification. This approach represents a valuable tool to investigate the role of PTMs crosstalk in protein function and cellular processes while it is less suitable for dynamic regulation of protein modification. In contrast with chemical synthesis of PTMs, such approaches benefit of a high level of spatial control over the engineered modification, allowing the user to modify proteins with exquisite spatial resolution at the intracellular level.

Directed evolution is also a powerful tool for engineering protein domain specificity over cognate target residues or for developing enzymes with different activity (Figure 3b). An example comes from Cai et al. who in 2015 showed how directed evolution of the plant enzyme Monolignol 4-OMethyltransferase could be used to change its substrate specificity with consequent ability to improve lignin digestion and conversion. ${ }^{120}$ More recently, Dai et al. engineered the histidine methyltransferase SETD3, changing its target specificity from histidine to lysine. ${ }^{121}$ By comparing the active site of SETD3 
with the ones of the lysine methyltransferases SETD6 and LSMT, the authors individuated two amino acids, Asn55 and Trp273, responsible for histidine specificity. When these were mutated to phenylalanine and alanine respectively, the mutant protein showed a marked increase in catalytic efficiency in lysine methylation compared to the wild type protein. These approaches provide mechanistic information about the respective enzymes function, but also can be applied to assist in vivo engineering of biological systems by tailoring natural enzymatic processes. Given the extent of all methylation reactions throughout the cell, the potential to exploit similar systems is very high.

For sure, more knowledge is still to come to elucidate the mechanisms underlying the role of nonhistone protein methylation in pathway regulation and deeper understanding is needed for us to be able to exploit the full potential of this key regulatory mechanism for engineering applications. It is overwhelmingly clear however that non-histone protein methylation is an essential and wide-spread regulatory mechanism within the cell. The dynamic and specific interplay of methyltransferases and demethylases, methylated residues and other PTMs can allow subtle regulation of protein-protein interactions, increasing the potential complexity within engineered systems.

a

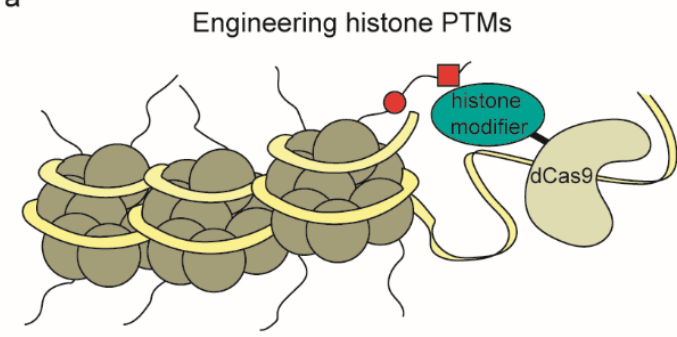

Engineering PTMs using genetic code expansion b

Engineering writers specificity

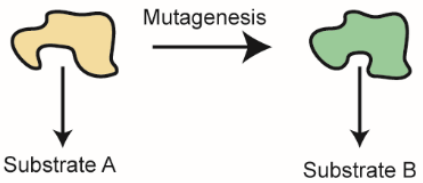

d Engineering PTMs using trans-protein splicing

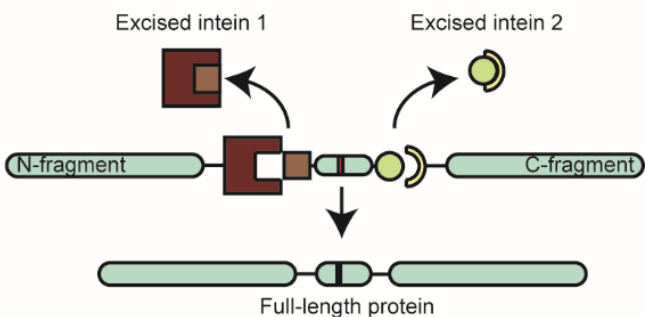

Figure 3. Engineering PTMs. a) Histone proteins can be appended with PTMs by directing the activity of histone modifiers fused to customisable DNA-binding proteins (such as dCas9) at defined genomic location. b) The specificity of PTMs writers can also be engineered through directed evolution or rational engineering approaches.c) Genetic code expansion can mediate the incorporation of custom amino acids into proteins. In this case amber and/or frameshift suppressor tRNA charged with the modified residue are used to introduce the desired modifications, following appropriate engineering of the protein-coding sequence. d) Trans-protein splicing where inteinextein pairs are used to mediate the splicing between the heterologously-expressed C-and $\mathrm{N}$ fragments of the protein, and its chemically synthesised central fragment carrying the desired modification(s). ${ }^{118}$

\section{Conclusion}


Non-histone protein methylation is involved in a wide variety of cellular functions, affecting the functionality of diverse protein-substrates. Whilst there is much progress is the proteome-wide identification of protein methylation, more work needs to be done, particularly in the detection and analysis of methyl-lysine PTMs. As our knowledge of the diverse functions of protein methylation increases, the significance of its role in disease and its potential for therapy become more apparent. Bioengineering has revealed insights into other PTMs, yet its application to the study and exploitation of non-histone protein methylation remains in its infancy. If we were able to easily engineer targeted methylation of non-histone proteins, we can increase the capacity to engineer protein function in critical biological pathways. This will be important not only to shed light on many undisclosed protein regulation mechanisms, but it will also be an invaluable asset for integrating transcriptional and posttranslational regulation for the engineering of increasingly complex genetic circuits.

\section{Acknowledgements}

\section{Funding and additional information}

CRUK C33325/A236190. Infrastructure support for this work was provided by the NIHR Imperial Biomedical Research Centre.

\section{Conflict of interest}

The authors declare that they have no conflicts of interest with the contents of this article.

\section{References}

(1) Ambler, R. P.; Rees, M. W. Epsilon-N-Methyl-Lysine in Bacterial Flagellar Protein. Nature 1959, 184, 56-57.

(2) Murray, K. The Occurrence of I $\varepsilon$-N-Methyl Lysine in Histones. Biochemistry 1964, 3 (1), 10 15. https://doi.org/10.1021/bi00889a003.

(3) Paik, W. K.; Kim, S. Protein Methylase I. Purification and Properties of the Enzyme. J. Biol. Chem. 1968, 243 (9), 2108-2114.

(4) Paik, W. K.; Paik, D. C.; Kim, S. Historical Review: The Field of Protein Methylation. Trends in Biochemical Sciences. Trends Biochem Sci March 2007, pp 146-152. https://doi.org/10.1016/j.tibs.2007.01.006.

(5) Murn, J.; Shi, Y. The Winding Path of Protein Methylation Research: Milestones and New Frontiers. Nature Reviews Molecular Cell Biology. Nature Publishing Group August 2017, pp 517-527. https://doi.org/10.1038/nrm.2017.35.

(6) Shi, Y.; Lan, F.; Matson, C.; Mulligan, P.; Whetstine, J. R.; Cole, P. A.; Casero, R. A.; Shi, Y. Histone Demethylation Mediated by the Nuclear Amine Oxidase Homolog LSD1. Cell 2004, 119 (7), 941-953. https://doi.org/10.1016/j.cell.2004.12.012.

(7) Chang, B.; Chen, Y.; Zhao, Y.; Bruick, R. K. JMJD6 Is a Histone Arginine Demethylase. Science (80-. ). 2007, 318 (5849), 444-447. https://doi.org/10.1126/science.1145801.

(8) Unoki, M.; Masuda, A.; Dohmae, N.; Arita, K.; Yoshimatsu, M.; Iwai, Y.; Fukui, Y.; Ueda, K.; Hamamoto, R.; Shirakawa, M.; et al. Lysyl 5-Hydroxylation, a Novel Histone Modification, by Jumonji Domain Containing 6 (JMJD6). J. Biol. Chem. 2013, 288 (9), 60536062. https://doi.org/10.1074/jbc.M112.433284.

(9) Zhang, M.; Xu, J. Y.; Hu, H.; Ye, B. C.; Tan, M. Systematic Proteomic Analysis of Protein Methylation in Prokaryotes and Eukaryotes Revealed Distinct Substrate Specificity.

Proteomics 2018, 18 (1), 1-13. https://doi.org/10.1002/pmic.201700300. 
(10) Reimand, J.; Wagih, O.; Bader, G. D. Evolutionary Constraint and Disease Associations of Post-Translational Modification Sites in Human Genomes. PLoS Genet. 2015, 11 (1), e1004919. https://doi.org/10.1371/journal.pgen.1004919.

(11) Hornbeck, P. V.; Zhang, B.; Murray, B.; Kornhauser, J. M.; Latham, V.; Skrzypek, E. PhosphoSitePlus, 2014: Mutations, PTMs and Recalibrations. Nucleic Acids Res. 2015, 43 (D1), D512-D520. https://doi.org/10.1093/nar/gku1267.

(12) Evich, M.; Stroeva, E.; Zheng, Y. G.; Germann, M. W. Effect of Methylation on the SideChain PKa Value of Arginine. Protein Sci. 2016, 25 (2), 479-486.

https://doi.org/10.1002/pro.2838.

(13) Qamar, S.; Wang, G.; Randle, S. J.; Ruggeri, F. S.; Varela, J. A.; Lin, J. Q.; Phillips, E. C.; Miyashita, A.; Williams, D.; Ströhl, F.; et al. FUS Phase Separation Is Modulated by a Molecular Chaperone and Methylation of Arginine Cation- $\pi$ Interactions. Cell 2018, 173 (3), 720-734.e15. https://doi.org/10.1016/j.cell.2018.03.056.

(14) Ferrari, L.; Stucchi, R.; Konstantoulea, K.; van de Kamp, G.; Kos, R.; Geerts, W. J. C.; van Bezouwen, L. S.; Förster, F. G.; Altelaar, M.; Hoogenraad, C. C.; et al. Arginine $\pi$-Stacking Drives Binding to Fibrils of the Alzheimer Protein Tau. Nat. Commun. 2020, 11 (1), 571. https://doi.org/10.1038/s41467-019-13745-7.

(15) Raposo, A. E.; Piller, S. C. Protein Arginine Methylation: An Emerging Regulator of the Cell Cycle. Cell Div. 2018, 13 (1), 1-16. https://doi.org/10.1186/s13008-018-0036-2.

(16) Lee, S.-Y.; Jang, C.; Lee, K.-A. Polo-Like Kinases (Plks), a Key Regulator of Cell Cycle and New Potential Target for Cancer Therapy. Dev. Reprod. 2014, 18 (1), 65-71. https://doi.org/10.12717/dr.2014.18.1.065.

(17) Seki, A.; Coppinger, J. A.; Du, H.; Jang, C. Y.; Yates, J. R.; Fang, G. Plk1- and $\beta$-TrCPDependent Degradation of Bora Controls Mitotic Progression. J. Cell Biol. 2008, 181 (1), 6578. https://doi.org/10.1083/jcb.200712027.

(18) Li, W.; Wang, H. Y.; Zhao, X.; Duan, H.; Cheng, B.; Liu, Y.; Zhao, M.; Shu, W.; Mei, Y.; Wen, Z.; et al. A Methylation-Phosphorylation Switch Determines Plk1 Kinase Activity and Function in DNA Damage Repair. Sci. Adv. 2019, 5 (3), eaau7566. https://doi.org/10.1126/sciadv.aau7566.

(19) Feldman, M.; Vershinin, Z.; Goliand, I.; Elia, N.; Levy, D. The Methyltransferase SETD6 Regulates Mitotic Progression through PLK1 Methylation. Proc. Natl. Acad. Sci. U. S. A. 2019, 116 (4), 1235-1240. https://doi.org/10.1073/pnas.1804407116.

(20) Campaner, S.; Spreafico, F.; Burgold, T.; Doni, M.; Rosato, U.; Amati, B.; Testa, G. The Methyltransferase Set7/9 (Setd7) Is Dispensable for the P53-Mediated DNA Damage Response In Vivo. Mol. Cell 2011, 43 (4), 681-688.

https://doi.org/10.1016/j.molcel.2011.08.007.

(21) Vadnais, C.; Chen, R.; Fraszczak, J.; Hamard, P. J.; Manfredi, J. J.; Möröy, T. A Novel Regulatory Circuit between P53 and GFI1 Controls Induction of Apoptosis in T Cells. Sci. Rep. 2019, 9 (1), 1-9. https://doi.org/10.1038/s41598-019-41684-2.

(22) Zheng, S.; Moehlenbrink, J.; Lu, Y.-C.; Zalmas, L.-P.; Sagum, C. A.; Carr, S.; McGouran, J. F.; Alexander, L.; Fedorov, O.; Munro, S.; et al. Arginine Methylation-Dependent ReaderWriter Interplay Governs Growth Control by E2F-1. Mol. Cell 2013, 52 (1), 37-51. https://doi.org/10.1016/j.molcel.2013.08.039.

(23) Roworth, A. P.; Carr, S. M.; Liu, G.; Barczak, W.; Miller, R. L.; Munro, S.; Kanapin, A.; Samsonova, A.; La Thangue, N. B. Arginine Methylation Expands the Regulatory Mechanisms and Extends the Genomic Landscape under E2F Control. Sci. Adv. 2019, 5 (6), 1- 
14. https://doi.org/10.1126/sciadv.aaw4640.

(24) Sasikumar, A. N.; Perez, W. B.; Kinzy, T. G. The Many Roles of the Eukaryotic Elongation Factor 1 Complex. Wiley Interdisciplinary Reviews: RNA. NIH Public Access July 2012, pp 543-555. https://doi.org/10.1002/wrna.1118.

(25) Hamey, J. J.; Wilkins, M. R. Methylation of Elongation Factor 1A: Where, Who, and Why? Trends Biochem. Sci. 2018, 43 (3), 211-223.

https://doi.org/https://doi.org/10.1016/j.tibs.2018.01.004 211.

(26) Malecki, J.; Aileni, V. K.; Ho, A. Y. Y.; Schwarz, J.; Moen, A.; Sørensen, V.; Nilges, B. S.; Jakobsson, M. E.; Leidel, S. A.; Falnes, P. Ø. The Novel Lysine Specific Methyltransferase METTL21B Affects MRNA Translation through Inducible and Dynamic Methylation of Lys165 in Human Eukaryotic Elongation Factor 1 Alpha (EEF1A). Nucleic Acids Res. 2017, 45 (8), 4370-4389. https://doi.org/10.1093/nar/gkx002.

(27) Hamey, J. J.; Wienert, B.; Quinlan, K. G. R.; Wilkins, M. R. METTL21B Is a Novel Human Lysine Methyltransferase of Translation Elongation Factor 1A: Discovery by CRISPR/Cas9 Knockout. Mol. Cell. Proteomics 2017, 16 (12), 2229-2242. https://doi.org/10.1074/mcp.M116.066308.

(28) Fang, L.; Zhang, L.; Wei, W.; Jin, X.; Wang, P.; Tong, Y.; Li, J.; Du, J. X.; Wong, J. A Methylation-Phosphorylation Switch Determines Sox2 Stability and Function in ESC Maintenance or Differentiation. Mol. Cell 2014, 55 (4), 537-551. https://doi.org/10.1016/j.molcel.2014.06.018.

(29) Zhang, C.; Hoang, N.; Leng, F.; Saxena, L.; Lee, L.; Alejo, S.; Qi, D.; Khal, A.; Sun, H.; Lu, F.; et al. LSD1 Demethylase and the Methyl-Binding Protein PHF20L1 Prevent SET7 Methyltransferase- Dependent Proteolysis of the Stem-Cell Protein SOX2. J. Biol. Chem. 2018, 293 (10), 3663-3674. https://doi.org/10.1074/jbc.RA117.000342.

(30) Zhang, C.; Leng, F.; Saxena, L.; Hoang, N.; Yu, J.; Alejo, S.; Lee, L.; Qi, D.; Lu, F.; Sun, H.; et al. Proteolysis of Methylated SOX2 Protein Is Regulated by L3MBTL3 and CRL4DCAF5 Ubiquitin Ligase. J. Biol. Chem. 2019, 294 (2), 476-489.

https://doi.org/10.1074/jbc.RA118.005336.

(31) Rendón, O. Z.; Neiva, S.; Sasarman, F.; Shoubridge, E. A. The Arginine Methyltransferase NDUFAF7 Is Essential for Complex I Assembly and Early Vertebrate Embryogenesis. Hum. Mol. Genet. 2014, 23 (19), 5159-5170. https://doi.org/10.1093/hmg/ddu239.

(32) Sharma, L.; Lu, J.; Bai, Y. Mitochondrial Respiratory Complex I: Structure, Function and Implication in Human Diseases. Curr. Med. Chem. 2009, 16 (10), 1266-1277. https://doi.org/10.2174/092986709787846578.

(33) Rhein, V. F.; Carroll, J.; Ding, S.; Fearnley, I. M.; Walker, J. E. NDUFAF7 Methylates Arginine 85 in the NDUFS2 Subunit of Human Complex I. J. Biol. Chem. 2013, 288 (46), 33016-33026. https://doi.org/10.1074/jbc.M113.518803.

(34) Małecki, J. M.; Willemen, H. L. D. M.; Pinto, R.; Ho, A. Y. Y.; Moen, A.; Eijkelkamp, N.; Falnes, P. Ø. Human FAM173A Is a Mitochondrial Lysine-Specific Methyltransferase That Targets Adenine Nucleotide Translocase and Affects Mitochondrial Respiration. J. Biol. Chem. 2019, 294 (31), 11654-11664. https://doi.org/10.1074/jbc.RA119.009045.

(35) Małecki, J. M.; Willemen, H. L. D. M.; Pinto, R.; Ho, A. Y. Y.; Moen, A.; Kjønstad, I. F.; Burgering, B. M. T.; Zwartkruis, F.; Eijkelkamp, N.; Falnes, P. Ø. Lysine Methylation by the Mitochondrial Methyltransferase FAM173B Optimizes the Function of Mitochondrial ATP Synthase. J. Biol. Chem. 2019, 294 (4), 1128-1141. https://doi.org/10.1074/jbc.RA118.005473. 
(36) Rhein, V. F.; Carroll, J.; Ding, S.; Fearnley, I. M.; Walker, J. E. Human METTL12 Is a Mitochondrial Methyltransferase That Modifies Citrate Synthase. FEBS Lett. 2017, 591 (12), 1641-1652. https://doi.org/10.1002/1873-3468.12649.

(37) Yan, W.; Liang, Y.; Zhang, Q.; Wang, D.; Lei, M.; Qu, J.; He, X.; Lei, Q.; Wang, Y. Arginine Methylation of SIRT7 Couples Glucose Sensing with Mitochondria Biogenesis. EMBO Rep. 2018, 19 (12). https://doi.org/10.15252/embr.201846377.

(38) Johnson, P.; Harris, C. I.; Perry, S. V. 3-Methylhistidine in Actin and Other Muscle Proteins. Biochem. J. 1967, 105 (1), 361-370. https://doi.org/10.1042/bj1050361.

(39) Terman, J. R.; Kashina, A. Post-Translational Modification and Regulation of Actin. Curr. Opin. Cell Biol. 2013, 25 (1), 30-38. https://doi.org/10.1016/j.ceb.2012.10.009.

(40) Dominguez, R.; Holmes, K. C. Actin Structure and Function. 2011. https://doi.org/10.1146/annurev-biophys-042910-155359.

(41) Kwiatkowski, S.; Seliga, A. K.; Vertommen, D.; Terreri, M.; Ishikawa, T.; Grabowska, I.; Tiebe, M.; Teleman, A. A.; Jagielski, A. K.; Veiga-da-Cunha, M.; et al. SETD3 Protein Is the Actin-Specific Histidine N-Methyltransferase. Elife 2018, 7. https://doi.org/10.7554/eLife.37921.

(42) Wilkinson, A. W.; Diep, J.; Dai, S.; Liu, S.; Ooi, Y. S.; Song, D.; Li, T.-M.; Horton, J. R.; Zhang, X.; Liu, C.; et al. SETD3 Is an Actin Histidine Methyltransferase That Prevents Primary Dystocia. Nature 2019, 565 (7739), 372-376. https://doi.org/10.1038/s41586-0180821-8.

(43) Guo, Q.; Liao, S.; Kwiatkowski, S.; Tomaka, W.; Yu, H.; Wu, G.; Tu, X.; Min, J.; Drozak, J.; $\mathrm{Xu}, \mathrm{C}$. Structural Insights into SETD3-Mediated Histidine Methylation on $\beta$-Actin. Elife 2019, 8. https://doi.org/10.7554/eLife.43676.

(44) Dai, S.; Horton, J. R.; Woodcock, C. B.; Wilkinson, A. W.; Zhang, X.; Gozani, O.; Cheng, X. Structural Basis for the Target Specificity of Actin Histidine Methyltransferase SETD3. Nat. Commun. 2019, 10 (1), 3541. https://doi.org/10.1038/s41467-019-11554-6.

(45) Abaev-Schneiderman, E.; Admoni-Elisha, L.; Levy, D. SETD3 Is a Positive Regulator of DNA-Damage-Induced Apoptosis. Cell Death Dis. 2019, 10 (2), 74. https://doi.org/10.1038/s41419-019-1328-4.

(46) Cheng, X.; Hao, Y.; Shu, W.; Zhao, M.; Zhao, C.; Wu, Y.; Peng, X.; Yao, P.; Xiao, D.; Qing, G.; et al. Cell Cycle-Dependent Degradation of the Methyltransferase SETD3 Attenuates Cell Proliferation and Liver Tumorigenesis. J. Biol. Chem. 2017, 292 (22), 9022-9033. https://doi.org/10.1074/jbc.M117.778001.

(47) Xiao, W.; Chen, X.; Liu, L.; Shu, Y.; Zhang, M.; Zhong, Y. Role of Protein Arginine Methyltransferase 5 in Human Cancers. Biomed. Pharmacother. 2019, 114 (January), 108790. https://doi.org/10.1016/j.biopha.2019.108790.

(48) He, A.; Shen, X.; Ma, Q.; Cao, J.; von Gise, A.; Zhou, P.; Wang, G.; Marquez, V. E.; Orkin, S. H.; Pu, W. T. PRC2 Directly Methylates GATA4 and Represses Its Transcriptional Activity. Genes Dev. 2012, 26 (1), 37-42. https://doi.org/10.1101/gad.173930.111.

(49) Mazur, P. K.; Reynoird, N.; Khatri, P.; Jansen, P. W. T. C.; Wilkinson, A. W.; Liu, S.; Barbash, O.; Van Aller, G. S.; Huddleston, M.; Dhanak, D.; et al. SMYD3 Links Lysine Methylation of MAP3K2 to Ras-Driven Cancer. Nature 2014, 510 (7504), 283-287. https://doi.org/10.1038/nature13320.

(50) Huang, J.; Perez-Burgos, L.; Placek, B. J.; Sengupta, R.; Richter, M.; Dorsey, J. A.; Kubicek, S.; Opravil, S.; Jenuwein, T.; Berger, S. L. Repression of P53 Activity by Smyd2-Mediated Methylation. Nature 2006, 444 (7119), 629-632. https://doi.org/10.1038/nature05287. 
(51) Kim, K. Y.; Wang, D.-H.; Campbell, M.; Huerta, S. B.; Shevchenko, B.; Izumiya, C.; Izumiya, Y. PRMT4-Mediated Arginine Methylation Negatively Regulates Retinoblastoma Tumor Suppressor Protein and Promotes E2F-1 Dissociation. Mol. Cell. Biol. 2014, 35 (1), 238-248. https://doi.org/10.1128/mcb.00945-14.

(52) Kunizaki, M.; Hamamoto, R.; Silva, F. P.; Yamaguchi, K.; Nagayasu, T.; Shibuya, M.; Nakamura, Y.; Furukawa, Y. The Lysine 831 of Vascular Endothelial Growth Factor Receptor 1 Is a Novel Target of Methylation by SMYD3. Cancer Res. 2007, 67 (22), 10759-10765. https://doi.org/10.1158/0008-5472.CAN-07-1132.

(53) Piao, L.; Kang, D.; Suzuki, T.; Masuda, A.; Dohmae, N.; Nakamura, Y.; Hamamoto, R. The Histone Methyltransferase SMYD2 Methylates PARP1 and Promotes Poly(ADP-

Ribosyl)Ation Activity in Cancer Cells. Neoplasia (United States) 2014, 16 (3), 257-264.e2. https://doi.org/10.1016/j.neo.2014.03.002.

(54) Zhu, Y.; He, X.; Lin, Y. C.; Dong, H.; Zhang, L.; Chen, X.; Wang, Z.; Shen, Y.; Li, M.; Wang, H.; et al. Targeting PRMT1-Mediated FLT3 Methylation Disrupts Maintenance of MLLRearranged Acute Lymphoblastic Leukemia. Blood 2019, 134 (15), 1257-1268. https://doi.org/10.1182/blood.2019002457.

(55) Hamamoto, R.; Toyokawa, G.; Nakakido, M.; Ueda, K.; Nakamura, Y. SMYD2-Dependent HSP90 Methylation Promotes Cancer Cell Proliferation by Regulating the Chaperone Complex Formation. Cancer Lett. 2014, 351 (1), 126-133. https://doi.org/10.1016/j.canlet.2014.05.014.

(56) Rodríguez-Paredes, M.; Lyko, F. The Importance of Non-Histone Protein Methylation in Cancer Therapy. Nat. Rev. Mol. Cell Biol. 2019, 20 (10), 569-570. https://doi.org/10.1038/s41580-019-0147-x.

(57) Copeland, R. A. Protein Methyltransferase Inhibitors as Precision Cancer Therapeutics: A Decade of Discovery. Philos. Trans. R. Soc. B Biol. Sci. 2018, 373 (1748). https://doi.org/10.1098/RSTB.2017.0080.

(58) Stein, E. M.; Garcia-Manero, G.; Rizzieri, D. A.; Tibes, R.; Berdeja, J. G.; Savona, M. R.; Jongen-Lavrenic, M.; Altman, J. K.; Thomson, B.; Blakemore, S. J.; et al. The DOT1L Inhibitor Pinometostat Reduces H3K79 Methylation and Has Modest Clinical Activity in Adult Acute Leukemia. Blood 2018, 131 (24), 2661-2669. https://doi.org/10.1182/blood-201712-818948.

(59) Marcos-Villar, L.; Nieto, A. The DOT1L Inhibitor Pinometostat Decreases the Host-Response against Infections: Considerations about Its Use in Human Therapy. Sci. Rep. 2019, 9 (1), 16862. https://doi.org/10.1038/s41598-019-53239-6.

(60) Zhu, B.; Chen, S.; Wang, H.; Yin, C.; Han, C.; Peng, C.; Liu, Z.; Wan, L.; Zhang, X.; Zhang, J.; et al. The Protective Role of DOT1L in UV-Induced Melanomagenesis. Nat. Commun. 2018, 9 (259). https://doi.org/10.1038/s41467-017-02687-7.

(61) Lattouf, H.; Poulard, C.; Le Romancer, M. PRMT5 Prognostic Value in Cancer. Oncotarget 2019, 10 (34), 3151-3153. https://doi.org/10.18632/oncotarget.26883.

(62) Jakobsson, M. E.; Moen, A.; Davidson, B.; Falnes, P. Hsp70 (HSPA1) Lysine Methylation Status as a Potential Prognostic Factor in Metastatic High-Grade Serous Carcinoma. PLoS One 2015, 10 (10). https://doi.org/10.1371/journal.pone.0140168.

(63) Illei, P. B.; Rusch, V. W.; Zakowski, M. F.; Ladanyi, M. Homozygous Deletion of CDKN2A and Codeletion of the Methylthioadenosine Phosphorylase Gene in the Majority of Pleural Mesotheliomas. Clin. Cancer Res. 2003, 9 (6), 2108-2113.

(64) Mavrakis, K. J.; Robert McDonald, E.; Schlabach, M. R.; Billy, E.; Hoffman, G. R.; DeWeck, 
A.; Ruddy, D. A.; Venkatesan, K.; Yu, J.; McAllister, G.; et al. Disordered Methionine Metabolism in MTAP/CDKN2A-Deleted Cancers Leads to Dependence on PRMT5. Science (80-. ). 2016, 351 (6278), 1208-1213. https://doi.org/10.1126/science.aad5944.

(65) Marjon, K.; Cameron, M. J.; Quang, P.; Clasquin, M. F.; Mandley, E.; Kunii, K.; McVay, M.; Choe, S.; Kernytsky, A.; Gross, S.; et al. MTAP Deletions in Cancer Create Vulnerability to Targeting of the MAT2A/PRMT5/RIOK1 Axis. Cell Rep. 2016, 15 (3), 574-587. https://doi.org/10.1016/j.celrep.2016.03.043.

(66) Kryukov, G. V.; Wilson, F. H.; Ruth, J. R.; Paulk, J.; Tsherniak, A.; Marlow, S. E.; Vazquez, F.; Weir, B. A.; Fitzgerald, M. E.; Tanaka, M.; et al. MTAP Deletion Confers Enhanced Dependency on the PRMT5 Arginine Methyltransferase in Cancer Cells. Science (80-. ). 2016, 351 (6278), 1214-1218. https://doi.org/10.1126/science.aad5214.

(67) Strobl, C. D.; Schaffer, S.; Haug, T.; Volkl, S.; Peter, K.; Singer, K.; Bottcher, M.; Mougiakakos, D.; Mackensen, A.; Aigner, M. Selective PRMT5 Inhibitors Suppress Human CD8p T Cells by Upregulation of P53 and Impairment of the Akt Pathway Similar to the Tumor Metabolite MTA. Mol. Cancer Ther. 2020, 19 (2), 409-419. https://doi.org/10.1158/1535-7163.MCT-19-0189.

(68) Gao, G.; Zhang, L.; Villarreal, O. D.; He, W.; Su, D.; Bedford, E.; Moh, P.; Shen, J.; Shi, X.; Bedford, M. T.; et al. PRMT1 Loss Sensitizes Cells to PRMT5 Inhibition. Nucleic Acids Res. 2019, 47 (10), 5038-5048. https://doi.org/10.1093/nar/gkz200.

(69) Chong, F. P.; Ng, K. Y.; Koh, R. Y.; Chye, S. M. Tau Proteins and Tauopathies in Alzheimer's Disease. Cell. Mol. Neurobiol. 2018, 38 (5), 965-980. https://doi.org/10.1007/s10571-0170574-1.

(70) Leulliot, N.; Quevillon-Cheruel, S.; Sorel, I.; De La Sierra-Gallay, I. L.; Collinet, B.; Graille, M.; Blondeau, K.; Bettache, N.; Poupon, A.; Janin, J.; et al. Structure of Protein Phosphatase Methyltransferase 1 (PPM1), a Leucine Carboxyl Methyltransferase Involved in the Regulation of Protein Phosphatase 2A Activity. J. Biol. Chem. 2004, 279 (9), 8351-8358. https://doi.org/10.1074/jbc.M311484200.

(71) Guénin, S.; Schwartz, L.; Morvan, D.; Steyaert, J. M.; Poignet, A.; Madelmont, J. C.; Demidem, A. PP2A Activity Is Controlled by Methylation and Regulates Oncoprotein Expression in Melanoma Cells: A Mechanism Which Participates in Growth Inhibition Induced by Chloroethylnitrosourea Treatment. Int. J. Oncol. 2008, 32 (1), 49-57.

(72) Park, H.-J.; Lee, K.-W.; Oh, S.; Yan, R.; Zhang, J.; Beach, T. G.; Adler, C. H.; Voronkov, M.; Braithwaite, S. P.; Stock, J. B.; et al. Protein Phosphatase 2A and Its Methylation Modulating Enzymes LCMT-1 and PME-1 Are Dysregulated in Tauopathies of Progressive Supranuclear Palsy and Alzheimer Disease. J. Neuropathol. Exp. Neurol. 2018, 77 (2), 139-148. https://doi.org/10.1093/jnen/nlx110.

(73) Thomas, S. N.; Funk, K. E.; Wan, Y.; Liao, Z.; Davies, P.; Kuret, J.; Yang, A. J. Dual Modification of Alzheimer's Disease PHF-Tau Protein by Lysine Methylation and Ubiquitylation: A Mass Spectrometry Approach. Acta Neuropathol. 2012, 123 (1), 105-117. https://doi.org/10.1007/s00401-011-0893-0.

(74) Funk, K. E.; Thomas, S. N.; Schafer, K. N.; Cooper, G. L.; Liao, Z.; Clark, D. J.; Yang, A. J.; Kuret, J. Lysine Methylation Is an Endogenous Post-Translational Modification of Tau Protein in Human Brain and a Modulator of Aggregation Propensity. Biochem. J. 2014, 462 (1), $77-$ 88. https://doi.org/10.1042/BJ20140372.

(75) Huseby, C. J.; Hoffman, C. N.; Cooper, G. L.; Cocuron, J. C.; Alonso, A. P.; Thomas, S. N.; Yang, A. J.; Kuret, J. Quantification of Tau Protein Lysine Methylation in Aging and Alzheimer's Disease. J. Alzheimer's Dis. 2019, 71 (3), 979-991. https://doi.org/10.3233/JAD190604. 
(76) Yamaguchi, A.; Kitajo, K. The Effect of PRMT1-Mediated Arginine Methylation on the Subcellular Localization, Stress Granules, and Detergent-Insoluble Aggregates of FUS/TLS. PLoS One 2012, 7 (11), e49267. https://doi.org/10.1371/journal.pone.0049267.

(77) Scheer, S.; Ackloo, S.; Medina, T. S.; Schapira, M.; Li, F.; Ward, J. A.; Lewis, A. M.; Northrop, J. P.; Richardson, P. L.; Kaniskan, H. Ü.; et al. A Chemical Biology Toolbox to Study Protein Methyltransferases and Epigenetic Signaling. Nat. Commun. 2019, 10 (1). https://doi.org/10.1038/s41467-018-07905-4.

(78) Sidoli, S.; Kori, Y.; Lopes, M.; Yuan, Z.-F.; Kim, H. J.; Kulej, K.; Janssen, K. A.; Agosto, L. M.; Cunha, J. P. C. da; Andrews, A. J.; et al. One Minute Analysis of 200 Histone Posttranslational Modifications by Direct Injection Mass Spectrometry. Genome Res. 2019, 29 (6), 978-987. https://doi.org/10.1101/GR.247353.118.

(79) Levy, D.; Liu, C. L.; Yang, Z.; Newman, A. M.; Alizadeh, A. A.; Utz, P. J.; Gozani, O. A Proteomic Approach for the Identification of Novel Lysine Methyltransferase Substrates. Epigenetics Chromatin 2011, 4 (1), 19. https://doi.org/10.1186/1756-8935-4-19.

(80) Hart-Smith, G.; Yagoub, D.; Tay, A. P.; Pickford, R.; Wilkins, M. R. Large Scale Mass Spectrometry-Based Identifications of Enzyme-Mediated Protein Methylation Are Subject to High False Discovery Rates. Mol. Cell. Proteomics 2016, 15 (3), 989-1006. https://doi.org/10.1074/mcp.M115.055384.

(81) Katsanovskaja, K.; Driver, T.; Pipkorn, R.; Edelson-Averbukh, M. Negative Ion Mode Collision-Induced Dissociation for Analysis of Protein Arginine Methylation. J. Am. Soc. Mass Spectrom. 2019, 30 (7), 1229-1241. https://doi.org/10.1007/s13361-019-02176-9.

(82) Hartel, N. G.; Liu, C. Z.; Graham, N. A. Improved Discrimination of Asymmetric and Symmetric Arginine Dimethylation by Optimization of the Normalized Collision Energy in Liquid Chromatography-Mass Spectrometry Proteomics. https://doi.org/10.1021/acs.jproteome.0c00116.

(83) Geoghegan, V.; Guo, A.; Trudgian, D.; Thomas, B.; Acuto, O. Comprehensive Identification of Arginine Methylation in Primary T Cells Reveals Regulatory Roles in Cell Signalling. Nat. Commun. 2015, 6, 6758. https://doi.org/10.1038/ncomms7758.

(84) Ong, S.-E.; Mittler, G.; Mann, M. Identifying and Quantifying in Vivo Methylation Sites by Heavy Methyl SILAC. Nat. Methods 2004, 1 (2), 119-126. https://doi.org/10.1038/nmeth715.

(85) Tay, A. P.; Geoghegan, V.; Yagoub, D.; Wilkins, M. R.; Hart-Smith, G. MethylQuant: A Tool for Sensitive Validation of Enzyme-Mediated Protein Methylation Sites from Heavy-Methyl SILAC Data. J. Proteome Res. 2018, 17 (1), 359-373. https://doi.org/10.1021/acs.jproteome.7b00601.

(86) Massignani, E.; Cuomo, A.; Musiani, D.; Jammula, S. G.; Pavesi, G.; Bonaldi, T. HmSEEKER: Identification of HmSILAC Doublets in MaxQuant Output Data. Proteomics 2019, 19 (5), 1800300. https://doi.org/10.1002/pmic.201800300.

(87) Chaudhari, M.; Thapa, N.; Roy, K.; Newman, R.; Saigo, H.; Dukka, B. K. C. DeepRMethylSite: A Deep Learning Based Approach for Prediction of Arginine Methylation Sites in Proteins. Mol. Omi. 2020. https://doi.org/10.1039/d0mo00025f.

(88) Guo, A.; Gu, H.; Zhou, J.; Mulhern, D.; Wang, Y.; Lee, K. A.; Yang, V.; Aguiar, M.; Kornhauser, J.; Jia, X.; et al. Immunoaffinity Enrichment and Mass Spectrometry Analysis of Protein Methylation. Mol. Cell. Proteomics 2014, 13 (1), 372-387. https://doi.org/10.1074/mcp.O113.027870.

(89) Cao, X.-J.; Arnaudo, A. M.; Garcia, B. A. Large-Scale Global Identification of Protein Lysine Methylation in Vivo. Epigenetics 2013, 8 (5), 477-485. https://doi.org/10.4161/epi.24547. 
(90) Cao, X.-J.; Garcia, B. A. Global Proteomics Analysis of Protein Lysine Methylation. Curr Protoc Protein Sci 2017, 86. https://doi.org/10.1002/cpps.16.

(91) Musiani, D.; Bok, J.; Massignani, E.; Wu, L.; Tabaglio, T.; Ippolito, M. R.; Cuomo, A.; Ozbek, U.; Zorgati, H.; Ghoshdastider, U.; et al. Proteomics Profiling of Arginine Methylation Defines PRMT5 Substrate Specificity. Sci. Signal. 2019, 12 (575), eaat8388. https://doi.org/10.1126/scisignal.aat8388.

(92) Larsen, S. C.; Sylvestersen, K. B.; Mund, A.; Lyon, D.; Mullari, M.; Madsen, M. V.; Daniel, J. A.; Jensen, L. J.; Nielsen, M. L. Proteome-Wide Analysis of Arginine Monomethylation Reveals Widespread Occurrence in Human Cells. Sci. Signal. 2016, 9 (443), rs9-rs9. https://doi.org/10.1126/scisignal.aaf7329.

(93) Hartel, N.; Chew, B.; Qin, J.; Xu, J.; Graham, N. Deep Protein Methylation Profiling by Combined Chemical and Immunoaffinity Approaches Reveals Novel PRMT1 Targets. bioRxiv 2019, 538686. https://doi.org/10.1101/538686.

(94) McCaddon, A.; Hudson, P. R. Methylation and Phosphorylation: A Tangled Relationship? Clin. Chem. 2007, 53 (6), 999-1000. https://doi.org/10.1373/clinchem.2007.086579.

(95) Lee, J.; Stock, J. Protein Phosphatase 2A Catalytic Subunit Is Methyl-Esterified at Its Carboxyl Terminus by a Novel Methyltransferase. J. Biol. Chem. 1993, 268 (26), 1919219195.

(96) Biggar, K. K.; Charih, F.; Liu, H.; Ruiz-Blanco, Y. B.; Stalker, L.; Chopra, A.; Connolly, J.; Adhikary, H.; Frensemier, K.; Hoekstra, M.; et al. Proteome-Wide Prediction of Lysine Methylation Leads to Identification of H2BK43 Methylation and Outlines the Potential Methyllysine Proteome. Cell Rep. 2020, 32 (2), 107896. https://doi.org/10.1016/j.celrep.2020.107896.

(97) Luo, M. Current Chemical Biology Approaches to Interrogate Protein Methyltransferases. ACS Chem. Biol. 2012, 7 (3), 443-463. https://doi.org/10.1021/cb200519y.

(98) Martin, J. L.; Mcmillan, F. M. SAM (Dependent) I AM: The S-AdenosylmethionineDependent Methyltransferase Fold. Proteins 2002, 12 (6), 783.

(99) Islam, K. The Bump-and-Hole Tactic: Expanding the Scope of Chemical Genetics. Cell Chem. Biol. 2018, 25 (10), 1171-1184. https://doi.org/10.1016/j.chembiol.2018.07.001.

(100) Zhang, J.; Zheng, Y. G. SAM/SAH Analogs as Versatile Tools for SAM-Dependent Methyltransferases. ACS Chem. Biol. 2016, 11 (3), 583-597.

https://doi.org/10.1021/acschembio.5b00812.

(101) Tekel, S. J.; Barrett, C.; Vargas, D.; Haynes, K. A. Design, Construction, and Validation of Histone-Binding Effectors in Vitro and in Cells. Biochemistry 2018, 57 (31), 4707-4716. https://doi.org/10.1021/acs.biochem.8b00327.

(102) Nyer, D. B.; Daer, R. M.; Vargas, D.; Hom, C.; Haynes, K. A. Regulation of Cancer Epigenomes with a Histone-Binding Synthetic Transcription Factor. npj Genomic Med. 2017, 2 (1), 1-10. https://doi.org/10.1038/s41525-016-0002-3.

(103) Haynes, K. A.; Silver, P. A. Synthetic Reversal of Epigenetic Silencing. J. Biol. Chem. 2011, 286 (31), 27176-27182. https://doi.org/10.1074/jbc.C111.229567.

(104) Gilbert, L. A.; Larson, M. H.; Morsut, L.; Liu, Z.; Brar, G. A.; Torres, S. E.; Stern-Ginossar, N.; Brandman, O.; Whitehead, E. H.; Doudna, J. A.; et al. CRISPR-Mediated Modular RNAGuided Regulation of Transcription in Eukaryotes. Cell 2013, 154 (2), 442. https://doi.org/10.1016/j.cell.2013.06.044.

(105) O’geen, H.; Ren, C.; Nicolet, C. M.; Perez, A. A.; Halmai, J.; Le, V. M.; Mackay, J. P.; 
Farnham, P. J.; Segal, D. J. DCas9-Based Epigenome Editing Suggests Acquisition of Histone Methylation Is Not Sufficient for Target Gene Repression. Nucleic Acids Res. 2017, 45 (17), 9901-9916. https://doi.org/10.1093/nar/gkx578.

(106) Kearns, N. A.; Pham, H.; Tabak, B.; Genga, R. M.; Silverstein, N. J.; Garber, M.; Maehr, R. Functional Annotation of Native Enhancers with a Cas9-Histone Demethylase Fusion. Nat. Methods 2015, 12 (5), 401-403. https://doi.org/10.1038/nmeth.3325.

(107) Cano-Rodriguez, D.; Gjaltema, R. A. F.; Jilderda, L. J.; Jellema, P.; Dokter-Fokkens, J.; Ruiters, M. H. J.; Rots, M. G. Writing of H3K4Me3 Overcomes Epigenetic Silencing in a Sustained but Context-Dependent Manner. Nat. Commun. 2016, 7. https://doi.org/10.1038/ncomms12284.

(108) Thakore, P. I.; D’Ippolito, A. M.; Song, L.; Safi, A.; Shivakumar, N. K.; Kabadi, A. M.; Reddy, T. E.; Crawford, G. E.; Gersbach, C. A. Highly Specific Epigenome Editing by CRISPR-Cas9 Repressors for Silencing of Distal Regulatory Elements. Nat. Methods 2015, 12 (12), 1143-1149. https://doi.org/10.1038/nmeth.3630.

(109) Lebar, T.; Lainšček, D.; Merljak, E.; Aupič, J.; Jerala, R. A Tunable Orthogonal Coiled-Coil Interaction Toolbox for Engineering Mammalian Cells. Nat. Chem. Biol. 2020, 16 (5), $513-$ 519. https://doi.org/10.1038/s41589-019-0443-y.

(110) Benedetti, L.; Barentine, A. E. S.; Messa, M.; Wheeler, H.; Bewersdorf, J.; De Camilli, P. Light-Activated Protein Interaction with High Spatial Subcellular Confinement. Proc. Natl. Acad. Sci. U. S. A. 2018, 115 (10), E2238-E2245. https://doi.org/10.1073/pnas.1713845115.

(111) Smadbeck, J.; Peterson, M. B.; Zee, B. M.; Garapaty, S.; Mago, A.; Lee, C.; Giannis, A.; Trojer, P.; Garcia, B. A.; Floudas, C. A. De Novo Peptide Design and Experimental Validation of Histone Methyltransferase Inhibitors. PLoS One 2014, 9 (2), 90095. https://doi.org/10.1371/journal.pone.0090095.

(112) Ai, H. W.; Lee, J. W.; Schultz, P. G. A Method to Site-Specifically Introduce Methyllysine into Proteins in E. Coli. Chem. Commun. 2010, 46 (30), 5506-5508. https://doi.org/10.1039/c0cc00108b.

(113) Groff, D.; Chen, P. R.; Peters, F. B.; Schultz, P. G. A Genetically Encoded $\varepsilon-N-M e t h y l$ Lysine in Mammalian Cells. ChemBioChem 2010, 11 (8), 1066-1068. https://doi.org/10.1002/cbic.200900690.

(114) Nguyen, D. P.; Garcia Alai, M. M.; Kapadnis, P. B.; Neumann, H.; Chin, J. W. Genetically Encoding Ne-Methyl-L-Lysine in Recombinant Histones. J. Am. Chem. Soc. 2009, 131 (40), 14194-14195. https://doi.org/10.1021/ja906603s.

(115) Nguyen, D. P.; Alai, M. M. G.; Virdee, S.; Chin, J. W. Genetically Directing $\varepsilon-N$, N-Dimethyll-Lysine in Recombinant Histones. Chem. Biol. 2010, 17 (10), 1072-1076. https://doi.org/10.1016/j.chembiol.2010.07.013.

(116) Tokuda, Y.; Watanabe, T.; Horiike, K.; Shiraga, K.; Abe, R.; Muranaka, N.; Hohsaka, T. Biosynthesis of Proteins Containing Modified Lysines and Fluorescent Labels Using NonNatural Amino Acid Mutagenesis. J. Biosci. Bioeng. 2011, 111 (4), 402-407. https://doi.org/10.1016/j.jbiosc.2010.12.012.

(117) Yanagisawa, T.; Takahashi, M.; Mukai, T.; Sato, S.; Wakamori, M.; Shirouzu, M.; Sakamoto, K.; Umehara, T.; Yokoyama, S. Multiple Site-Specific Installations of Ne-Monomethyl-LLysine into Histone Proteins by Cell-Based and Cell-Free Protein Synthesis. ChemBioChem 2014, 15 (12), 1830-1838. https://doi.org/10.1002/cbic.201402291.

(118) Khoo, K. K.; Galleano, I.; Gasparri, F.; Wieneke, R.; Harms, H.; Poulsen, M. H.; Chua, H. C.; Wulf, M.; Tampé, R.; Pless, S. A. Chemical Modification of Proteins by Insertion of Synthetic 
Peptides Using Tandem Protein Trans-Splicing. Nat. Commun. 2020, 11 (1), 1-10. https://doi.org/10.1038/s41467-020-16208-6.

(119) David, Y.; Vila-Perelló, M.; Verma, S.; Muir, T. W. Chemical Tagging and Customizing of Cellular Chromatin States Using Ultrafast Trans-Splicing Inteins. Nat. Chem. 2015, 7 (5), 394-402. https://doi.org/10.1038/nchem.2224.

(120) Cai, Y.; Bhuiya, M.-W.; Shanklin, J.; Liu, C.-J. Engineering a Monolignol 4-OMethyltransferase with High Selectivity for the Condensed Lignin Precursor Coniferyl Alcohol. J. Biol. Chem. 2015, 290 (44), 26715-26724. https://doi.org/10.1074/jbc.M115.684217.

(121) Dai, S.; Horton, J. R.; Wilkinson, A. W.; Gozani, O.; Zhang, X.; Cheng, X. An Engineered Variant of SETD3 Methyltransferase Alters Target Specificity from Histidine to Lysine Methylation. J. Biol. Chem. 2020, 295 (9), 2582-2589. https://doi.org/10.1074/jbc.RA119.012319. 